\title{
NOX2-dependent ATM kinase activation dictates pro-inflammatory macrophage phenotype and improves effectiveness to radiation therapy
}

\author{
Qiuji Wu ${ }^{1,2,3,4,5,6}$, Awatef Allouch ${ }^{1,2,3,4}$, Audrey Paoletti ${ }^{1,2,3,4}$, Celine Leteur ${ }^{2,3,4}$, Celine Mirjolet ${ }^{7}$, Isabelle Martins ${ }^{1,2,3,4}$, \\ Laurent Voisin ${ }^{1,2,3,4}$, Frédéric Law ${ }^{1,2,3,4}$, Haithem Dakhli ${ }^{1,2,3,4}$, Elodie Mintet ${ }^{1,2,3,4}$, Maxime Thoreau ${ }^{1,2,3,4}$, Zeinaf Muradova $^{1,2,3,4}$, \\ Mélanie Gauthier ${ }^{7}$, Olivier Caron $^{3}$, Fabien Milliat ${ }^{8}$, David M Ojcius ${ }^{9}$, Filippo Rosselli ${ }^{10}$, Eric Solary ${ }^{11}$, Nazanine Modjtahedi ${ }^{2,3,4}$, \\ Eric Deutsch ${ }^{2,3,4,12}$ and Jean-Luc Perfettini ${ }^{*, 1,2,3,4,12}$
}

\begin{abstract}
Although tumor-associated macrophages have been extensively studied in the control of response to radiotherapy, the molecular mechanisms involved in the ionizing radiation-mediated activation of macrophages remain elusive. Here we show that ionizing radiation induces the expression of interferon regulatory factor 5 (IRF5) promoting thus macrophage activation toward a proinflammatory phenotype. We reveal that the activation of the ataxia telangiectasia mutated (ATM) kinase is required for ionizing radiation-elicited macrophage activation, but also for macrophage reprogramming after treatments with $\gamma$-interferon, lipopolysaccharide or chemotherapeutic agent (such as cisplatin), underscoring the fact that the kinase ATM plays a central role during macrophage phenotypic switching toward a pro-inflammatory phenotype through the regulation of mRNA level and post-translational modifications of IRF5. We further demonstrate that NADPH oxidase 2 (NOX2)-dependent ROS production is upstream to ATM activation and is essential during this process. We also report that the inhibition of any component of this signaling pathway (NOX2, ROS and ATM) impairs pro-inflammatory activation of macrophages and predicts a poor tumor response to preoperative radiotherapy in locally advanced rectal cancer. Altogether, our results identify a novel signaling pathway involved in macrophage activation that may enhance the effectiveness of radiotherapy through the reprogramming of tumor-infiltrating macrophages.
\end{abstract}

Cell Death and Differentiation (2017) 24, 1632-1644; doi:10.1038/cdd.2017.91; published online 2 June 2017

Approximately, half of all cancer patients are treated with radiotherapy alone or in combination with chemotherapy. Although ionizing radiation (IR) directly causes senescence and death of tumor cells through the generation of reactive oxygen species (ROS) and DNA damage, ${ }^{1}$ recent studies underscore the fact that IR can also modulate immune cell functions and favor consequently the development of anticancer immunity. ${ }^{2,3}$ IR can induce the exposure of 'eat-me' signals (such as calreticulin) and the release of danger signals (such as ATP and HMGB1) by the irradiated dying tumor cells (also referred as immunogenic cell death), ${ }^{4}$ thus contributing to specific T-cell response by increasing the tumor antigen cross-presentation to dendritic cells ${ }^{5}$ and/or modifying the immunosuppressive microenvironment of tumors. ${ }^{6,7}$ IR can also control tumor immune response through the direct modulation of innate immune cell functions. Treatment with IR can modulate Langerhans cell functions and induce the accumulation of regulatory T cells into tumors. ${ }^{8}$ In addition, IR controls macrophage plasticity and programs tumorassociated macrophages (TAMs) toward a pro-inflammatory phenotype that orchestrates specific tumor immune response. ${ }^{9}$ However, microenvironment factors such as hypoxia may affect this IR-responsive macrophage activation program by favoring a pro-tumorigenic activation phenotype that is associated with tumor resistance. ${ }^{10}$ Nevertheless, the molecular mechanisms underlying IR-induced macrophage activation remain elusive.

TAMs represent a major cellular component of the tumor microenvironment. ${ }^{11}$ These macrophages derive from blood monocytes that, after their recruitment into tumors, differentiate and are activated in response to different environmental signals. Macrophages can be broadly classified as classically activated pro-inflammatory macrophages and alternatively activated protumorigenic macrophages. ${ }^{12}$ The interferon regulatory factor 5

${ }^{1}$ Cell Death and Aging Team, Gustave Roussy, 114 rue Edouard Vaillant, Villejuif F-94805, France; '2Laboratory of Molecular Radiotherapy, INSERM U1030, Gustave Roussy, 114 rue Edouard Vaillant, Villejuif F-94805, France; ${ }^{3}$ Gustave Roussy, 114 rue Edouard Vaillant, Villejuif F-94805, France; ${ }^{4}$ Université Paris Sud - Paris Saclay, 114 rue Edouard Vaillant, Villejuif F-94805, France; ${ }^{5}$ Department of Radiation and Medical Oncology, Zhongnan Hospital, Wuhan University, 169 Dong Hu Road, Wuhan 430071, China; ${ }^{6}$ Hubei Key Laboratory of Tumor Biological Behaviors, Zhongnan Hospital, Wuhan University, 169 Dong Hu Road, Wuhan 430071, China; ${ }^{7}$ Centre Georges François Leclerc, 1 rue du Pr Marion, Dijon F-21079, France; ${ }^{8}$ Laboratoire de Recherche en Radiobiologie et radiopathologie, Institut de Radioprotection et de Sureté Nucléaire, Fontenay-aux-Roses F-92262, France; ' Department of Molecular Cell Biology, Health Sciences Research Institute, University of California, Merced, CA 95343, USA; ${ }^{10}$ Laboratoire "Stabilité Génétique et Oncogenèse», CNRS - UMR 8200, 114 rue Edouard Vaillant, Villejuif F-94805, France and ${ }^{11}$ INSERM U1170, Gustave Roussy, 114 rue Edouard Vaillant, Villejuif F-94805, France

${ }^{*}$ Corresponding author: J-L Perfettini, Cell death and Aging Team, Gustave Roussy, 114 rue Edouard Vaillant, Villejuif F-94805, France. Tel: +33142115424 ; Fax: +33 1 42116665; E-mail: perfettini@orange.fr

${ }^{12} \mathrm{ED}$ and J-LP share senior coauthorship.

Received 31.8.16; revised 19.4.17; accepted 02.5.17; Edited by M Piacentini; published online 02.6.17 
(IRF5) was demonstrated to determine the pro-inflammatory macrophage phenotype ${ }^{13-15}$ along with other transcription factors (such as STAT1 and NF-kB), whereas IRF4, ${ }^{16}$ STAT6 $^{17}$ and $\mathrm{KLF} 4^{18}$ are key transcription factors required for the protumorigenic macrophage phenotype. Interferon gamma plus lipopolysaccharide (LPS) or tumor necrosis factor $a$ alone are known to induce classical macrophage activation that is characterized by an increased secretion of pro-inflammatory cytokines and chemokines (such as interleukin (IL)-1 $1 \beta$ ), an augmented production of inducible nitric oxide synthase (iNOS) $^{19}$ and of ROS. ${ }^{12}$ Conversely, glucocorticoids, IL-4/IL-13 and IL-10 drive macrophages toward the alternative activation program with a reduced production of pro-inflammatory cytokines but a higher level of anti-inflammatory IL-10, TGF- $\beta$, arginase, membrane scavenger and mannose receptors. Pro-inflammatory macrophages possess bactericidal and anti-tumoral activities, while pro-tumorigenic macrophages are associated with immune regulatory and tissue repair activities, highlighting the diversity of macrophage functions. In response to tumor microenvironment signals (such as hypoxia and tumor-derived lactate ${ }^{20,21}$ ), TAMs mainly adopt a pro-tumorigenic phenotype that contributes to tumor progression by promoting tumor growth, metastasis, angiogenesis and by suppressing immune responses. ${ }^{22-24}$ In addition, several studies have demonstrated that TAMs are also associated with treatment resistance and poor clinical outcomes in various cancer settings (such as lymphoma, melanoma, sarcomas and lung cancer ${ }^{25-28}$ ), making them attractive targets for the development of new anticancer strategies. ${ }^{29,30}$ In this context, a better understanding of the molecular basis of the IRmediated macrophage activation is needed for the improvement of the efficacy of radiotherapy. In this study, we explored the molecular mechanisms involved in IR-induced macrophage reprogramming.

\section{Results}

Cell-autonomous activation of macrophages after ionizing radiation. Considering that immune cells may influence the functional reprogramming of macrophages, ${ }^{29}$ we first analyzed IR-mediated macrophage activation using human colon tumor xenografts in immunodeficient mice. HCT116 cells were subcutaneously inoculated into the right flanks of athymic nude mice. Seven days after inoculation, the irradiation of the palpable tumor mass with a single dose of 20 Gy resulted in significant tumor growth inhibition, as compared to the controls (Figure 1a). After 29 days, the residual irradiated tumors did not show any increase of the

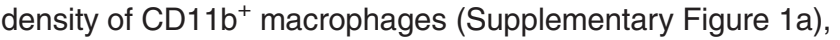
but revealed an increased frequency of $\mathrm{CD}_{11 \mathrm{~b}^{+}}$macrophages that expressed iNOS (iNOS ${ }^{+} \mathrm{CD}_{11 \mathrm{~b}^{+}}$) (Figure 1b). We detected a significant accumulation of iNOS $^{+} \mathrm{CD} 11 \mathrm{~b}^{+}$ macrophages in irradiated tumors as compared with non-irradiated ones (Figure 1c). The accumulation of iNOS $^{+} \mathrm{CD}_{11 \mathrm{~b}^{+}}$TAMs positively correlated with tumor response to IR, confirming as previously published that the presence of iNOS $^{+} /$pro-inflammatory phenotype macrophages in irradiated tumors is required for the modification of tumor microenvironment and tumor regression. Of note, previous reports characterized this process in conditions of relatively low-dose IR (2 Gy) exposure.6,9 Our observation that TAMs exhibited an increased phosphorylation of the histone variant $\mathrm{H} 2 \mathrm{AX}$ (also known as $\gamma$-H2AX) on serine 139 (Figures 1d and e) underlined an unsuspected link between DNA damage response and macrophage activation. To check the possibility whether IR can directly target and activate macrophages, we irradiated human THP1 macrophages with a single dose of 2 Gy and analyzed, by fluorescence microscopy and flow cytometry, the expression levels of iNOS and $\gamma$-H2AX. The increase in iNOS and $\gamma$-H2AX expression levels in irradiated THP1 macrophages (Figures 1f-h, Supplementary Figures $1 \mathrm{~b}$ and $1 \mathrm{c}$ ) revealed that IR could directly target macrophages to promote their activation toward a pro-inflammatory phenotype. We then analyzed by immunoblot the expression of a central transcription factor involved in macrophage activation, the IRF5 ${ }^{15}$ and observed that after single radiation doses of 2 and $4 \mathrm{~Gy}$, phorbol-12-myristate-13-acetate (PMA)-differentiated human THP1 macrophages (Figure 1i), human primary monocyte-derived macrophages (hMDM) (Figure 1j) and murine RAW264.7 macrophages (Figure 1k) exhibited an enhanced expression of IRF5 (Figures 1i-k). In addition, through immunoprecipitation experiments, we observed that after 2 Gy irradiation of RAW264.7 macrophages the proinflammatory transcription factor IRF5 was phosphorylated on serine (Figure 11). We also demonstrated that after, respectively, 96 and $12 \mathrm{~h}$ of irradiation, hMDM (Figure $1 \mathrm{~m}$ ) and murine RAW264.7 macrophages (Figure 1n) released two pro-inflammatory cytokines IL-1 $\beta$ and IL-8. To further complete the cytokine profile analysis, we determined the transcription levels of pro- and anti-inflammatory cytokines and observed increased mRNA expression of proinflammatory cytokines (such as TNF- $a$, IFN- $\gamma$, IL- 6 or IL-23) in 2 Gy-irradiated PMA-differentiated human THP1 macrophages (Figures 10-r) and detected an increased secretion of pro-inflammatory cytokines by $4 \mathrm{~Gy}$-irradiated hMDM (including TNF- $a$, IFN- $\gamma$, IL-6 and IL-8) as compared with controls (Figures 1s, t and Supplementary Figures $1 \mathrm{~d}-1 \mathrm{~g}$ ). Altogether, these results indicate that IR can promote a cell-autonomous activation of macrophages toward a pro-inflammatory phenotype.

ATM-mediated DNA damage response regulates the transcription of IRF5 in response to ionizing radiation. To further characterize the molecular mechanisms involved in IR-elicited macrophage activation, we first studied the induction of DNA damage-associated signaling pathways. Fifteen minutes after single radiation dose of $2 \mathrm{~Gy}$, PMAdifferentiated human THP1 macrophages (Figures 2a-c) exhibited a strong nuclear accumulation of $\mathrm{y}-\mathrm{H}_{2} \mathrm{AX}^{+}$foci (Figures $2 \mathrm{a}$ and $\mathrm{b}$ ) and of 53BP1 ${ }^{+}$foci (Figures $2 \mathrm{a}$ and $\mathrm{c}$ ) that could still be detected $6 \mathrm{~h}$ after exposure (Figures $2 \mathrm{~b}$ and $\mathrm{c}$ ), revealing the fact that DNA double-strand breaks are produced in response to IR. One hour after single radiation dose of 2 Gy, murine RAW264.7 macrophages also displayed increased $\gamma-\mathrm{H}_{2} \mathrm{AX}{ }^{+}$foci (Figures $2 \mathrm{~d}$ and e). Considering that the kinase ATM (mutated in the inherited recessive autosomal disease ataxia telangiectasia) is the major kinase involved in the phosphorylation of $\mathrm{H} 2 \mathrm{AX}$ (on serine 139), ${ }^{31}$ we evaluated the role of ATM in the activation of macrophages in response 
to IR. We observed that the vast majority of 2 Gy-irradiated murine RAW264.7 macrophages exhibited the activating auto-phosphorylation of ATM on serine 1981 (ATMS1918*) 1 -h post irradiation (Figures $2 d$ and $\mathrm{f}$ ). These results that were confirmed by immunoblots (Figures $2 \mathrm{~g}$ and $\mathrm{h}$ ) and flow cytometry (Supplementary Figure $2 \mathrm{2a}$ ) revealed also the positive correlation between ATMS1981* and IRF5 expression when hMDM (Figure $2 \mathrm{~g}$ ) or murine RAW264.7 macro- phages (Figure 2h) were irradiated with single doses of 2, 4 and 8 Gy. In addition, we detected an increase of ATMS1981* in $\mathrm{CD}^{+} 8^{+}$macrophages that were found in tumor samples after radiotherapy of rectal cancer patients (Figures $2 \mathrm{i}$ and $\mathrm{j}$ ), as compared to unirradiated patients. The ATMS1918* phosphorylation was positively correlated with the increased frequencies of tumor-associated $\mathrm{iNOS}^{+} \mathrm{CD} 68^{+}$macrophages that have been detected 6 weeks after radiotherapy
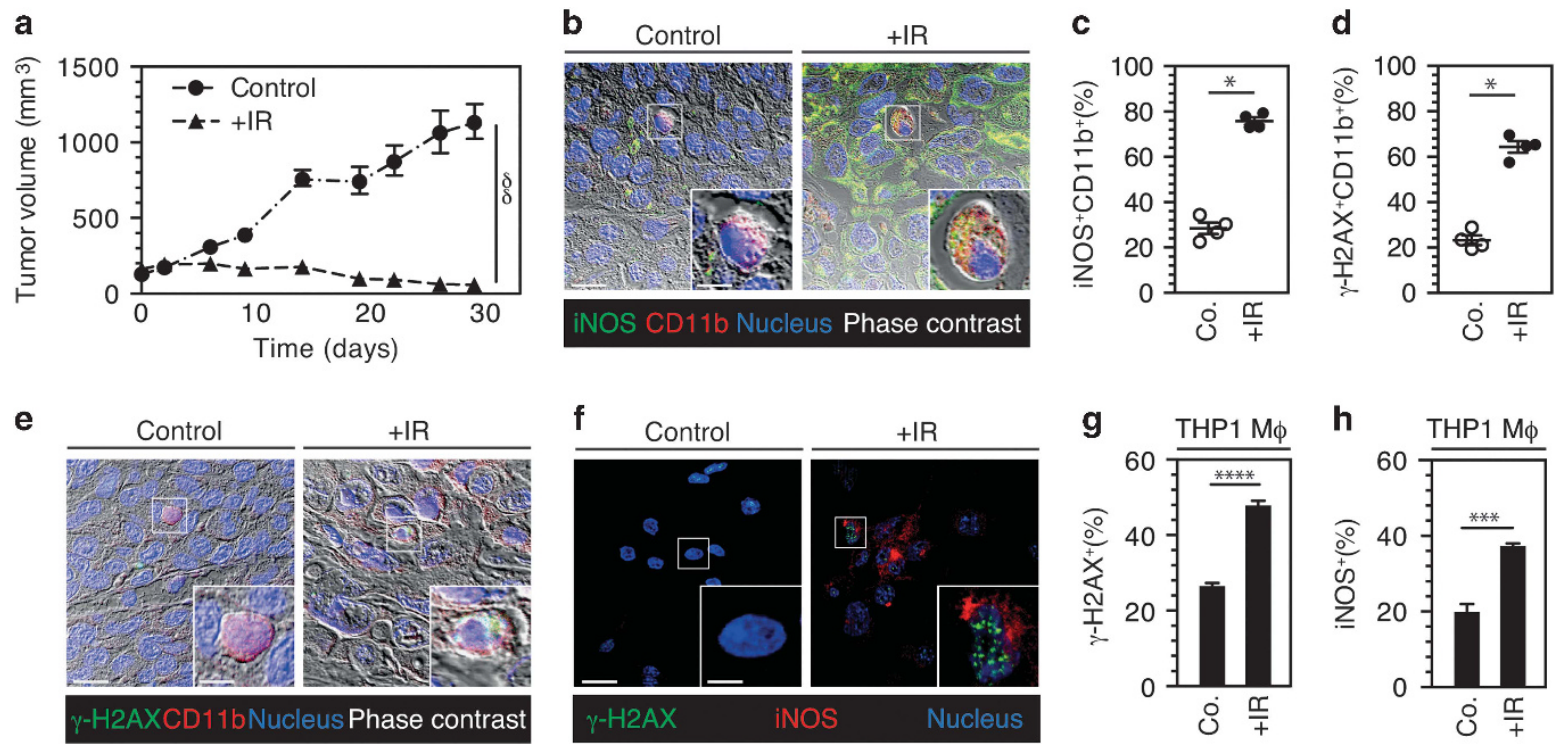

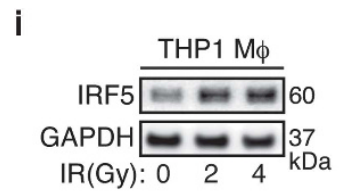

j

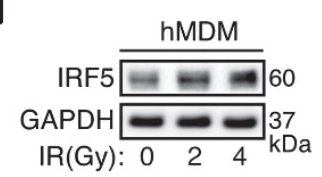

K

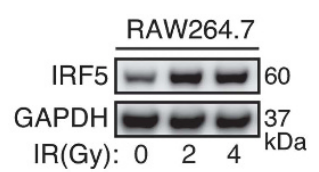

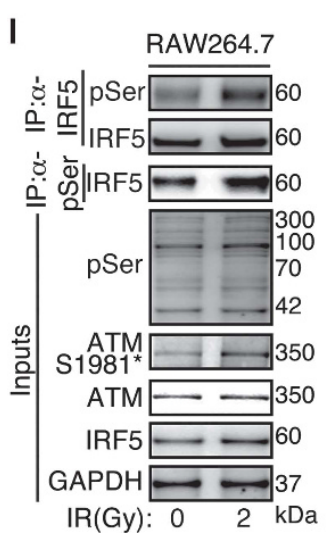

m

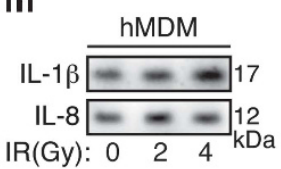

n

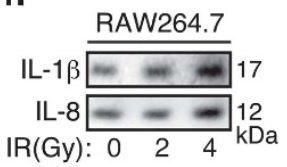

o

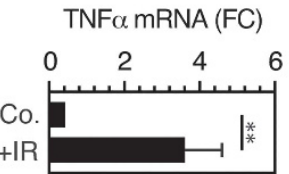

p IFN $\gamma$ mRNA (FC)

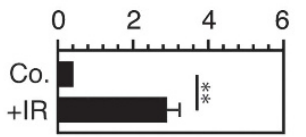

q IL-6 mRNA (FC)

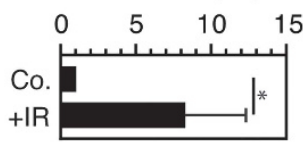

$r \quad$ IL-23 mRNA(FC)

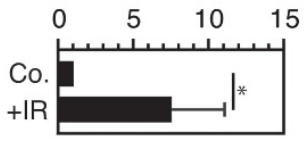

$\mathbf{S}$

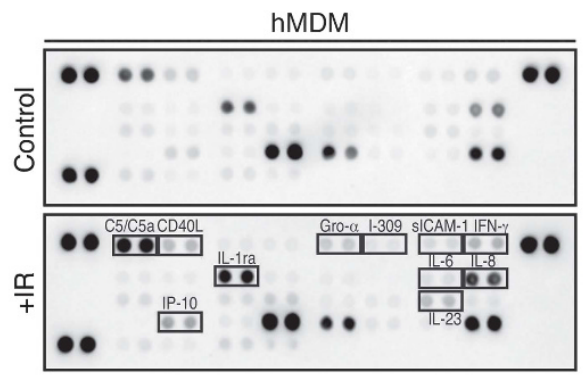

t

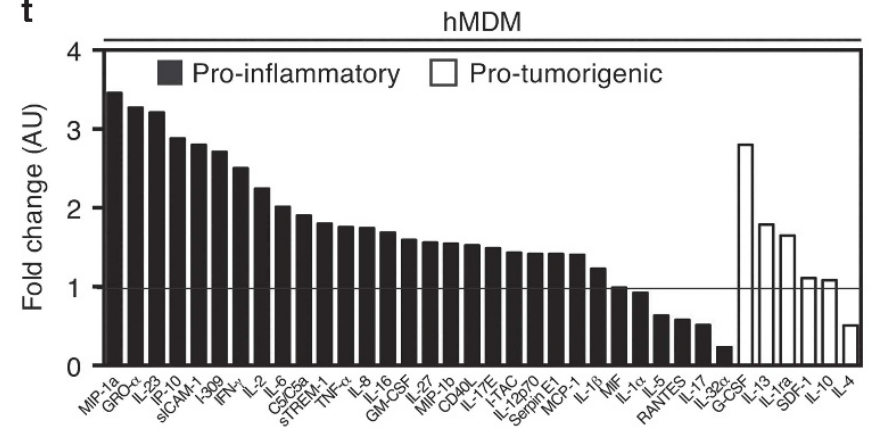


(Figures $2 \mathrm{k}$ and $\mathrm{I}$ ), demonstrating that the kinase ATM was sustainably activated in macrophages after radiotherapy. We next investigated the impact of ATM inactivation on IRinduced macrophage activation. The depletion of ATM by means of specific small interfering RNA (Figure $2 \mathrm{~m}$ ) or pharmacological inhibition with KU55933 (Figure 2n, Supplementary Figures $2 \mathrm{~b}-2 \mathrm{~d}$ and $2 \mathrm{i}$ ) impaired $\mathrm{y}-\mathrm{H} 2 \mathrm{AX}$ and ATMS1981* phosphorylation and the upregulation of IRF5 expression that was detected, respectively, 6 and $96 \mathrm{~h}$ after 2 and $4 \mathrm{~Gy}$ single-dose irradiation of murine RAW264.7 macrophages (Figure 2m, Supplementary Figures $2 b-2 d$ and $2 \mathrm{i}$ ) or hMDM (Figure $2 \mathrm{n}$ ) without altering macrophage viability (Supplementary Figures $2 \mathrm{e}-2 \mathrm{~h}$ ). We also observed that an enhancement of ATM activation through the pharmacological inhibition of poly(ADP-ribose)polymerase (PARP) with Olaparib further enhanced the inflammatory macrophage activation elicited by IR (as revealed by the increased expression of IRF5 (Figure 20)). Finally, we demonstrated that ATM regulated the expression of IRF5 at transcriptional level (as shown by quantitative real-time (RT) PCR (Figure $2 p$ and Supplementary Figure 2j)), confirming thus the central role of the kinase ATM during IR-mediated activation of macrophages toward a pro-inflammatory phenotype.

The kinase ATM dictates classical macrophage activation. In order to check whether the activation of ATM was a common feature of various pro-inflammatory macrophage activation programs, we analyzed the presence of DNA damage-associated nuclear foci in response to classical macrophage activators. ${ }^{32}$ Using confocal microscopy, we detected an accumulation of ATMS1981 ${ }^{*+}$ and $\gamma-\mathrm{H}_{2} \mathrm{AX}{ }^{+}$foci in the nuclei of murine RAW264.7 macrophages that were treated for $24 \mathrm{~h}$ with recombinant murine IFN- $\gamma(\mathrm{mIFN}-\gamma)$ or LPS (Figures $3 a-c)$. Using immunoblots, we also observed that the activation of ATM was concomitant with an increased expression of IRF5 in the PMA-differentiated THP1 macrophages (Figures 3d and f) or murine RAW264.7 macrophages (Figure 3e) stimulated with human or murine IFN- $\gamma$ (Figures $3 d$ and e) or LPS (Figure 3f). As expected, treatments of these macrophages with some other DNA strand break inducers (such as Cisplatin (Figures 3a-c, g and $\mathrm{h}$ ) or neocarzinostatin (NCZ) (Figure 3i)) or modulators of DNA repair (such as Olaparib (Figure $3 \mathrm{j}$ )), not only activated ATM but also increased IRF5 expression (Figures $3 g-j$ ). The results that are observed in absence of macrophage cytotoxicity (Supplementary Figure 3a) suggest that the DNA damage response signaling pathway might be a common pathway involved in classical macrophage activation. Moreover, we demonstrated that the pharmacological inhibition (Figures $3 \mathrm{k}$ and I) and the specific depletion (Figures $3 \mathrm{~m}$ and $n$ ) of ATM inhibited the increase of IRF5 expression that we previously detected after the treatment of PMA-differentiated THP1 macrophages (Figure 3k), murine RAW264.7 macrophages (Figures $3 \mathrm{l}$ and $\mathrm{m}$ ) or hMDM (Figure 3n) with human or murine IFN- $\gamma$ (Figures $3 k-n$ ) without impacting macrophage viability (Supplementary Figure $3 b$ ), confirming the essential role of the kinase ATM in classical macrophage activation.

ROS production induces ATMS1981* phosphorylation and IRF5 expression during macrophage activation. Considering that ROS have been involved in both ATM activation and macrophage differentiation, ${ }^{33,34}$ we investigated the role of ROS production during macrophage activation. Using flow cytometry to detect the conversion of the 2,7-dichlorohydro fluorescein diacetate $\left(\mathrm{H}_{2} \mathrm{DCFDA}\right)$ into 2,7-dichlorohydro fluorescein (DCF) when ROS are produced, we evaluated the ability of murine RAW264.7 macrophages to generate ROS following IR or mIFN- $\gamma$ treatment and revealed that both these treatments induced ROS production (Figures 4a-d). Importantly, we demonstrated that the N-acetyl cysteine (NAC) and the superoxide dismutase (SOD) mimetic $\mathrm{Mn}(\mathrm{III})$ tetrakis (4-benzoic acid) (MnTBAP) that blunted the ROS production (Figures $4 a-d$ ), inhibited also ATMS1981* (Figures $4 \mathrm{e}-\mathrm{g}$ ) and reduced the increased expression of IRF5 (Figures 4e-g) that we observed after treatment with IR (Figures $4 \mathrm{e}$ and $\mathrm{g}$ ) or mIFN- $\gamma$ (Figure 4f) of RAW264.7 macrophages (Figures 4e and $f$ ) or treatment with IR of PMA-differentiated THP1 macrophages (Figure $4 \mathrm{~g}$ ) without impacting macrophage

\footnotetext{
Figure 1 Irradiation activates macrophages toward pro-inflammatory phenotype.(a) Colorectal HCT116 cells were injected subcutaneously $\left(4 \times 10^{6}\right.$ cells per mouse) into immunodeficient mice and tumor growth was monitored. Results are expressed as mean value \pm S.E.M. $P$-value $\left({ }^{\delta \delta} P<0.01\right)$ was calculated by means of two-way ANOVA test. (b-e) Representative confocal micrographs and frequencies of iNOS $\mathrm{CD}^{+} 1 \mathrm{~b}^{+}(\mathbf{b}, \mathbf{c})$ or $\gamma-\mathrm{H}_{2 \mathrm{AX}} \mathrm{CD}_{11 \mathrm{~b}^{+}}(\mathbf{d}, \mathbf{e})$ tumor-associated macrophages detected in absence or after $20 \mathrm{~Gy}$ single-dose irradiation are shown (scale bar, $20 \mu \mathrm{m}$ ). Representative iNOS ${ }^{+} \mathrm{CD}_{11} \mathrm{~b}^{+}$or $\gamma-\mathrm{H} 2 \mathrm{AX} \mathrm{X}^{+} \mathrm{CD} 11 \mathrm{~b}^{+}$macrophages are shown in inserts (scale bar, $5 \mu \mathrm{m}$ ). Results are expressed as mean value \pm S.E.M. $P$-value $\left({ }^{*} P<0.05\right)$ was calculated using Mann-Whitney $U$-test. $(\mathbf{f}-\mathbf{h})$ Representative confocal micrographs and frequencies of phorbol-12myristate-13-acetate (PMA)-differentiated human THP1 macrophages showing $\gamma$-H2AX+ nuclear foci (f, $\mathbf{g}$ ) or expressing iNOS (iNOS ${ }^{+}$) (ff, $\mathbf{h}$ ) in control cells or $24 \mathrm{~h}$ after 2 Gy irradiation are shown (scale bar, $20 \mu \mathrm{m}$ ). Representative $\gamma-\mathrm{H}_{2} \mathrm{AX} \mathrm{X}^{+}$nuclear foci or iNOS expressing macrophages are shown in inserts (scale bar, $5 \mu \mathrm{m}$ ). Results are expressed as mean value \pm S.E.M. $P$-values ${ }^{* * \star} P<0.001,{ }^{* * \star *} P<0.0001$ ) were calculated using unpaired Student's $t$-test. (i-k) IRF5 expression after, respectively, 96,96 and 6 h culture of PMA-differentiated human THP1 macrophages (i), hMDM (j) or murine RAW264.7 macrophages (k) that have been irradiated (or not) with indicated doses. Representative immunoblots are shown. GAPDH is used as loading control. (I) Murine RAW264.7 macrophages that have been irradiated (or not) with 2 Gy were immunoprecipitated $6 \mathrm{~h}$ post irradiation for IRF5 and phopsho-serine (pSer), and analyzed for IRF5 and pSer expressions. Inputs were analyzed for IRF5, pSer, ATMS1981*, ATM and GAPDH. (m, $\mathbf{n}$ ) Detection of IL-1 $\beta$ and IL-8 release in the supernatants of hMDM $(\mathbf{m})$ or murine RAW264.7 macrophages $(\mathbf{n})$ that have been irradiated (or not) with indicated doses. Representative immunoblots are shown. (0-r) TNF $\alpha$, IFN $\gamma$, IL-6 and IL-23 mRNA expressions on PMA-differentiated THP1 macrophages that have been irradiated (or not) with 2 Gy were determined by quantitative real-time PCR. Results are expressed as mean value \pm S.E.M. and represented as fold change as compared to controls. $P$-values $\left({ }^{*} P<0.05,{ }^{* *} P<0.01\right.$ ) were calculated using unpaired Student's $t$-test. (s, t) Detection of cytokine secretion in the supernatants of hMDMs that have been treated (or not) with 4 Gy irradiation. Array images were captured following 1-10 min exposures to peroxidase substrate (s). Relative levels of cytokines detected in the supernatants of irradiated macrophages as compared to those detected in non-irradiated macrophages are revealed as fold change of arbitrary units. Pro-inflammatory and pro-tumorigenic cytokines and chemokines are indicated $(\mathrm{t})$. Data are representative of three independent experiments performed with primary human macrophages obtained from three healthy representative donors
} 
a

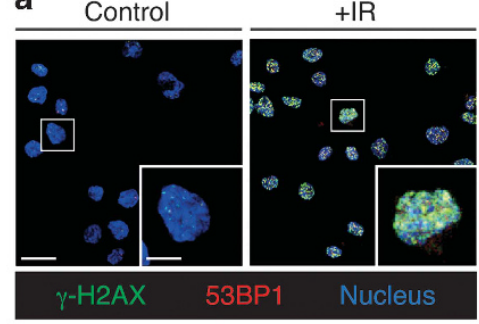

d

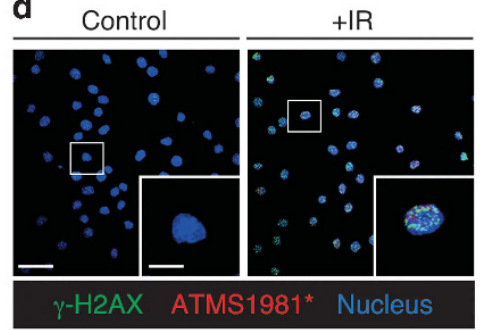

i Control
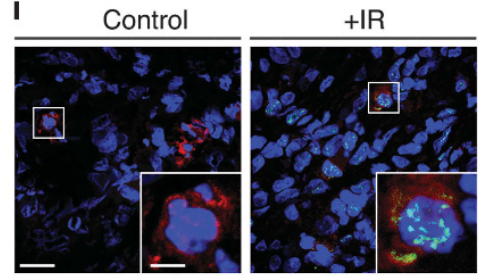

ATMS1981* CD68 b

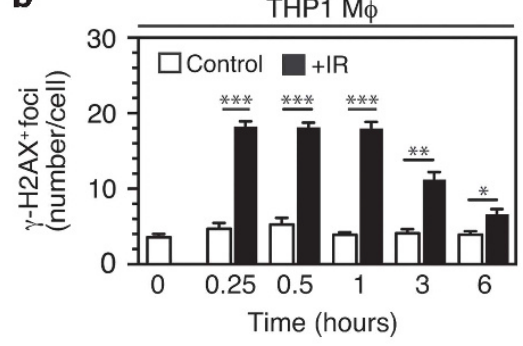

c

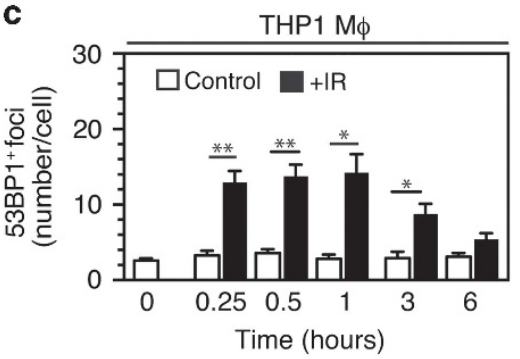

e
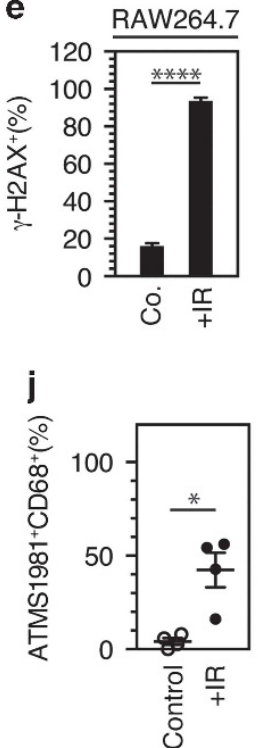

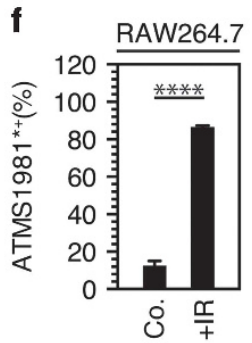

k

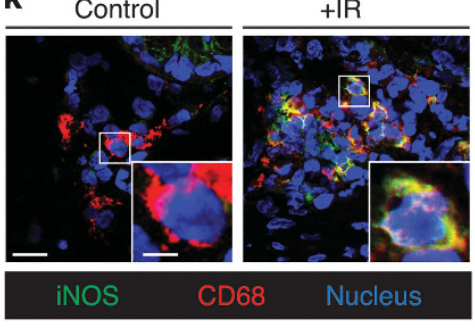

h ${ }_{\text {RAW264.7 }}$

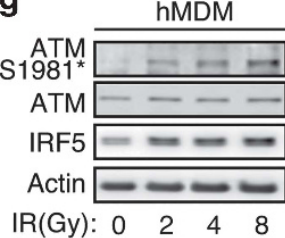

I

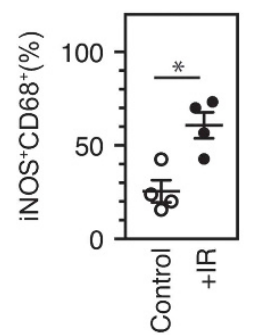

0

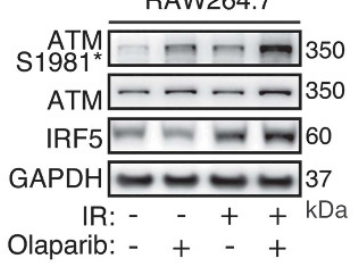

p

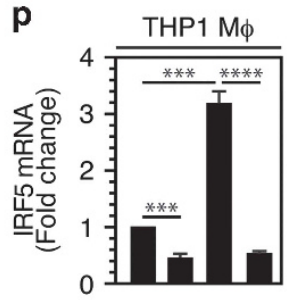

IR (Gy): - - 22

SiRNA Co.: + - + -

SIRNA ATM: - + - +

Figure 2 ATM activation controls IRF5 transcriptional expression and IR-induced pro-inflammatory macrophage phenotype.(a) Representative confocal micrographs of phorbol-12-myristate-13-acetate (PMA)- differentiated human THP1 macrophages showing $\gamma-\mathrm{H}_{2} \mathrm{AX}^{+}$or 53BP ${ }^{+}$foci following 2 Gy single-dose irradiation are shown (scale bar, $20 \mu \mathrm{m})$. Scale bar of inserts is $5 \mu \mathrm{m}$. (b, c) Frequencies of $\gamma-\mathrm{H}_{2} \mathrm{AX}^{+}$(b) or $53 \mathrm{BP}^{+}$(c) nuclear foci in PMA-differentiated human THP1 macrophages after $2 \mathrm{~Gy}$ single-dose irradiation are shown at indicated times. (d-f) Representative confocal micrographs and frequencies of murine RAW264.7 macrophages showing $\gamma$-H2AX ${ }^{+}$nuclear foci (d, e) or AMTS1981* phosphorylation (ATMS1981 $\left.{ }^{*+}\right)(\mathbf{d}, \mathrm{f})$, in control cells or $1 \mathrm{~h}$ after 2 Gy single-dose irradiation are shown (scale bar, $20 \mu \mathrm{m}$ ). Representative $\gamma$-H2AX ${ }^{+}$nuclear foci and ATMS1981 ${ }^{\star *}$ macrophages are shown in inserts (scale bar, $5 \mu \mathrm{m}$ ). Results are expressed as mean value \pm S.E.M. $P$-values $\left({ }^{*} P<0.05,{ }^{* *} P<0.01,{ }^{* \star *} P<0.001,{ }^{* * * *} P<0.0001\right)$ were calculated using unpaired Student's t-test. $(\mathbf{g}, \mathbf{h})$ ATMS1981*, ATM and IRF5 expression after, respectively, 96 and $6 \mathrm{~h}$ culture of hMDM (g) or murine RAW264.7 macrophages $(\mathrm{h})$ that have been irradiated (or not) with indicated doses are determined. Representative immunoblots are shown. Actin is used as loading control. (i-l) Representative confocal micrographs and frequencies of $\mathrm{ATMS} 1981^{\star+} \mathrm{CD}_{68}{ }^{+}(\mathrm{i}, \mathrm{j})$ or iNOS${ }^{+} \mathrm{CD} 68^{+}(\mathrm{k}, \mathrm{l})$ macrophages that have been detected in absence or after $45 \mathrm{~Gy}$ total dose of fractionated irradiation on tumor samples obtained from locally advanced rectal cancer patients are shown (scale bar, $20 \mu \mathrm{m} ;$ scale bar of insert, $5 \mu \mathrm{m}$ ). Results are expressed as mean value \pm S.E.M. $P$-value $\left({ }^{*} P<0.05\right)$ was calculated using Mann-Whitney $U$-test. $(\mathbf{m}, \mathbf{n})$ ATMS1981*, ATM and IRF5 expression after, respectively, 6 and $96 \mathrm{~h}$ culture of murine RAW264.7 macrophages that have been depleted for ATM (m) or hMDM that have been treated with $20 \mu$ M of KU55933 (n) and irradiated (or not) with 2 Gy ( $\mathbf{m}$ ) or 4 Gy (n) are shown. Representative immunoblots are shown. GAPDH (or actin) is used as loading control. (o) ATMS1981*, ATM and IRF5 expression after 6 h culture of murine RAW264.7 macrophages that have been treated with $10 \mu \mathrm{M}$ of Olaparib and irradiated (or not) with $2 \mathrm{~Gy}$ are shown. Representative immunoblots are shown. GAPDH is used as loading control. (p) IRF5 mRNA expression on PMA-differentiated human THP1 macrophages that have been depleted for ATM and irradiated (or not) with 2 Gy was determined by quantitative real-time PCR. Results are expressed as mean value \pm S.E.M. and represented as fold change as compared to controls. $P$-values $\left(^{* * \star} P<0.001\right.$ and ${ }^{* * \star *} P<0.0001$ ) were calculated using one-way ANOVA test. Quantification of western blot bands are shown in Supplementary Figure 4 
viability (Supplementary Figures $3 c$ and 3d). Altogether, these results indicate that by controlling the phosphorylation of ATM and the induction of the IRF5, ROS produced in the stimulated macrophages play a key role in the activation process.
The NADPH oxidase 2 is responsible for ROS production and ATM phosphorylation during macrophage activation. The NADPH oxidases are major regulated sources of ROS generation. ${ }^{35,36}$ To characterize mechanisms that are involved in ROS generation during macrophage activation, a

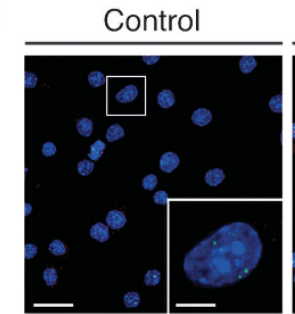

$\gamma$-H2AX
mIFN- $\gamma$

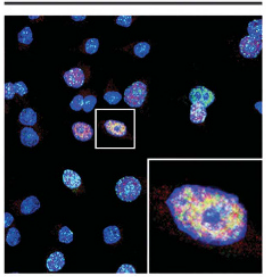

LPS

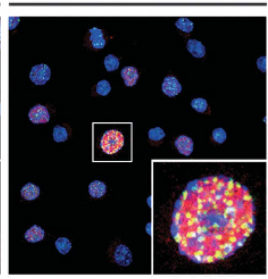

CDDP

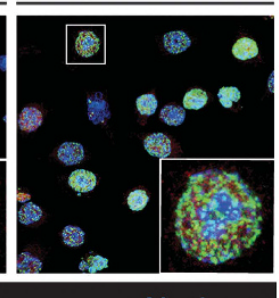

b

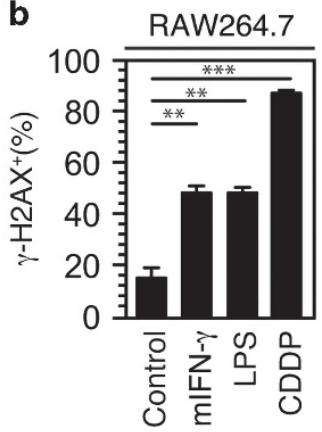

C

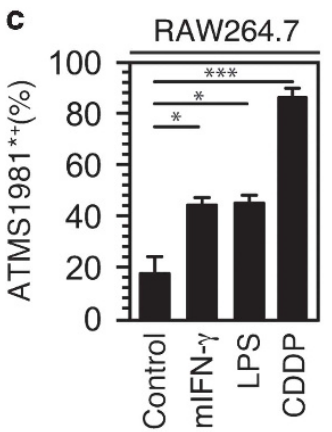

h

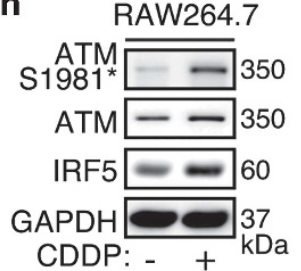

d

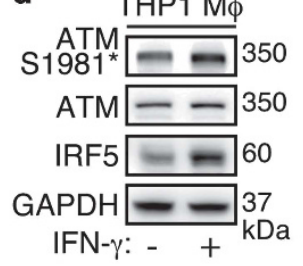

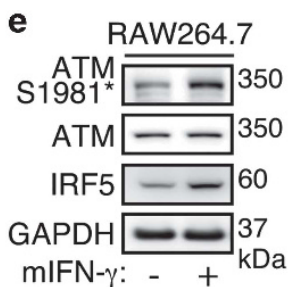

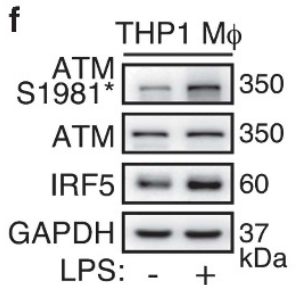

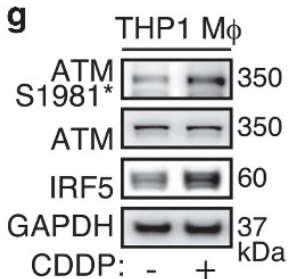

I

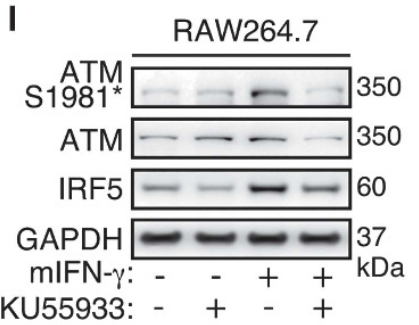

i RAW264.7

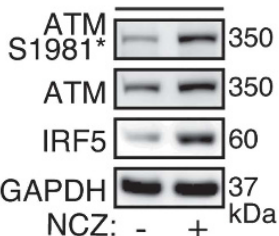

j

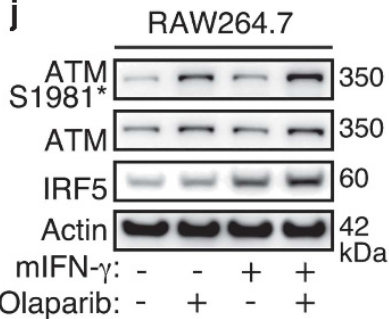

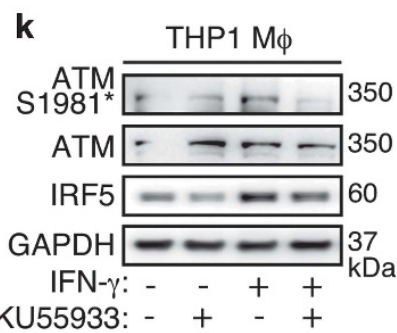

n

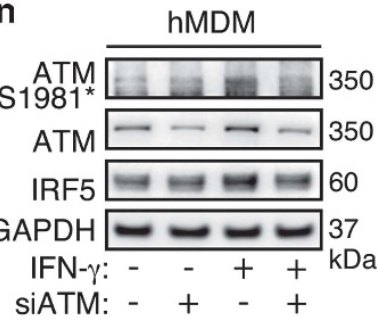

Figure 3 Classical macrophage activation is dependent on ATM. (a-c) Representative confocal micrographs and frequencies of murine RAW264.7 macrophages showing $\gamma$ $\mathrm{H} 2 \mathrm{AX}{ }^{+}$nuclear foci $(\mathbf{a}, \mathbf{b})$ or ATMS1981* phosphorylation (ATMS1981*+) $(\mathbf{a}, \mathbf{c})$ in control cells or after $24 \mathrm{~h}$ treatments with $20 \mathrm{ng} / \mathrm{ml}$ of recombinant murine IFN- $\gamma(\mathrm{mIFN}-\gamma)$, $100 \mathrm{ng} / \mathrm{ml}$ of lipopolysaccharide (LPS) or $10 \mu \mathrm{M}$ of cisplatinium (CDDP) are shown (scale bar, $20 \mu \mathrm{m}$ ). Representative macrophages with ATMS1981 $^{\star+}$ and $\gamma-\mathrm{H}_{2} \mathrm{AX} \mathrm{X}^{+}$nuclear foci are shown in inserts (scale bar, $5 \mu \mathrm{m}$ ). Results are expressed as mean value \pm S.E.M. $P$-values $\left({ }^{\star} P<0.05,{ }^{* \star} P<0.01,{ }^{* \star \star} P<0.001\right.$ ) were calculated using unpaired Student's $t$ test. (d-i) ATMS1981*, ATM and IRF5 expressions after $24 \mathrm{~h}$ culture of PMA-differentiated human THP1 macrophages $(\mathbf{d}, \mathbf{f}, \mathbf{g})$ or murine RAW264.7 macrophages (e, $\mathbf{h}$, i) that have been treated (or not) with $20 \mathrm{ng} / \mathrm{ml}$ of recombinant human IFN- $\gamma$ (IFN- $\gamma$ ) (d), $20 \mathrm{ng} / \mathrm{ml}$ of recombinant murine IFN- $\gamma(\mathrm{mlFN}-\gamma)(\mathbf{e}), 100 \mathrm{ng} / \mathrm{ml}$ of LPS (f), $10 \mu \mathrm{M}$ of CDDP (g, h) or $200 \mathrm{ng} / \mathrm{ml}$ of neocarzinostatin (NCZ) (i) are determined. Representative immunoblots are shown. GAPDH is used as loading control. (j-n) ATMS1981*, ATM and IRF5 expressions after, respectively, $24 \mathrm{~h}$ culture of murine RAW264.7 macrophages $(\mathrm{j}, \mathrm{I}, \mathrm{m})$, PMA-differentiated human THP1 macrophages $(\mathrm{\kappa})$ or hMDMs $(\mathrm{n})$ that have been incubated with $10 \mu \mathrm{M}$ of Olaparib (j), with $20 \mu \mathrm{M}$ of KU55933 (k, I) or depleted for ATM (m, n) and treated (or not) with $20 \mathrm{ng} / \mathrm{ml}$ mIFN- $($ for RAW264.7 macrophages) (j, I, m), $20 \mathrm{ng} / \mathrm{ml}$ human IFN- $\gamma$ (for PMA-differentiated human THP1 macrophages) ( $\kappa$ ) or $4 \mu \mathrm{g} / \mathrm{ml}$ of human IFN- $\gamma$ (for hMDM) (n) are evaluated. Representative immunoblots are shown. Actin (or GAPDH) is used as loading control. Quantification of western blot bands is shown in Supplementary Figure 4 


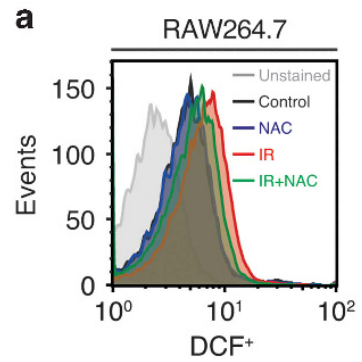

e

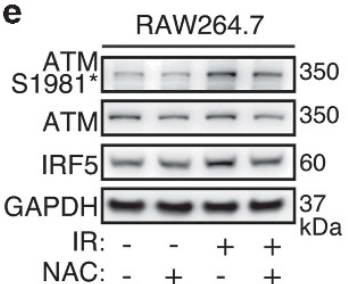

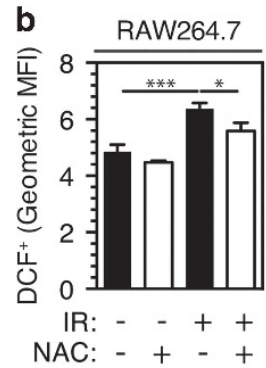
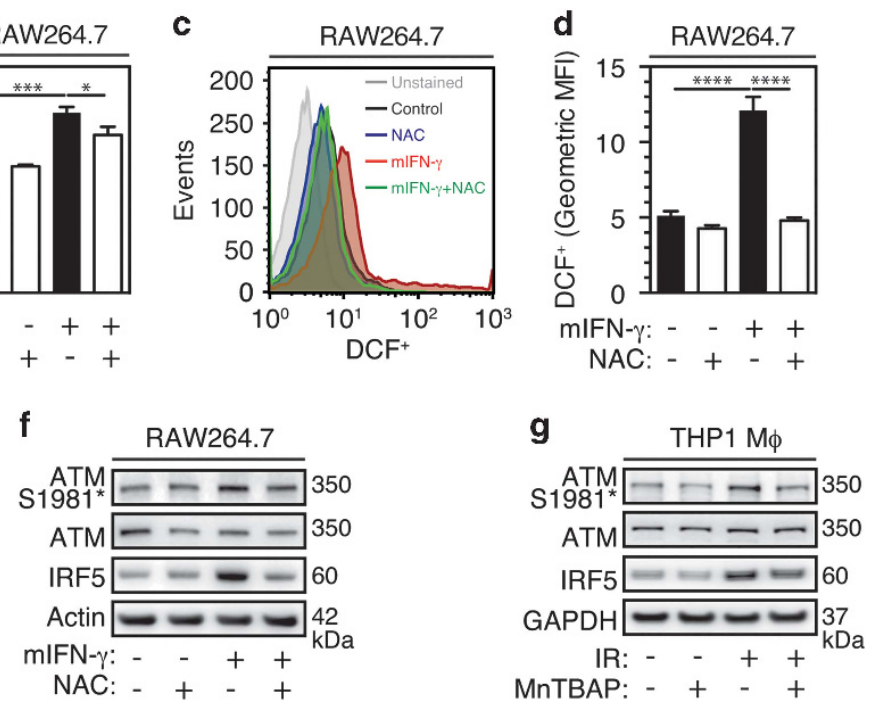

Figure 4 Reactive oxygen species are involved in IR-induced pro-inflammatory macrophage activation.(a-d) Murine RAW264.7 macrophages treated with $1 \mu \mathrm{g} / \mathrm{ml}$ of NAC were stimulated with 2 Gy single-dose irradiation $(\mathbf{a}, \mathbf{b})$ or $20 \mathrm{ng} / \mathrm{ml} \mathrm{mIFN}-\gamma(\mathbf{c}, \mathbf{d})$, stained with $\mathrm{H}_{2} \mathrm{DCFDA}$ and analyzed by flow cytometry. Representative flow cytometry analysis and quantifications of geometric mean fluorescence intensity (MFI) are shown. Data are presented as means \pm S.E.M. in $\mathbf{b}$ and $\mathbf{d}$ panels. Significances are ${ }^{*} P<0.05$, ${ }^{* \star *} P<0.001$ and ${ }^{* \star * *} P<0.0001$, and were obtained using one-way ANOVA test. (e, f) ATMS1981*, ATM and IRF5 expressions after, respectively, 6 and $24 \mathrm{~h}$ culture of murine RAW264.7 macrophages that have been incubated with $1 \mu \mathrm{g} / \mathrm{ml}$ of NAC and irradiated with 2 Gy single dose (e) or treated with $20 \mathrm{ng} / \mathrm{ml} \mathrm{mIFN- \gamma}$ (f) were determined. Representative immunoblots are shown. GAPDH (or actin) is used as loading control. (g) ATMS1981*, ATM and IRF5 expressions after $48 \mathrm{~h}$ culture of PMA-differentiated human THP1 macrophages that have been incubated with $10 \mu \mathrm{M}$ of MnTBAP and irradiated with 8 Gy single dose were determined. Representative immunoblots are shown. GAPDH is used as loading control. Quantification of western blot bands are shown in Supplementary Figure 5

we examined the role of NADPH oxidase 2 (NOX2), which is mainly expressed in macrophages and neutrophils. ${ }^{35,36}$ First, using immunoblots, we observed that NOX2 was upregulated after irradiation of PMA-differentiated THP1 macrophages (Figure $5 \mathrm{a}$ ) with single doses of 2 and $4 \mathrm{~Gy}$. These results were confirmed with the treatments of hMDM (Figure $5 b$ ), PMA-differentiated THP1 macrophages (Figure 5c) and RAW264.7 macrophages (Figures $5 \mathrm{~d}$ and $\mathrm{e}$ ) with human or murine IFN- $\gamma$ (Figures 5b, c and e) or IR (Figure 5d). NOX2 expression was also found increased in $\mathrm{CD} 68^{+}$macrophages that were detected in tumor samples obtained 6 weeks after radiotherapy of rectal cancer patients (Figures $5 f$ and $g$ ), as compared to biopsies obtained from the same patients before radiotherapy. Then, we evaluated the effect of the pharmacological NADPH oxidase inhibitor, diphenylene iodonium (DPI) on ROS production, ATMS1981* and IRF5 upregulation detected after the treatment of RAW264.7 macrophages with IR (Figures 5h, i and I) or mIFN- $\gamma$ (Figures 5j, k and m). We demonstrated that DPI impaired all events of the abovedescribed signaling cascade (Figures $5 \mathrm{~h}-\mathrm{m}$ ) without modifying macrophage viability (Supplementary Figures $3 e$ and $3 f$ ). In addition, as revealed by immunoblots, the depletion of NOX2 with specific small interfering RNA in irradiated (Figure $5 \mathrm{n}$ and Supplementary Figure $3 \mathrm{~g}$ ) or $\mathrm{mIFN}-\gamma$-treated (Figure 50 and Supplementary Figure 3h) RAW264.7 macrophages reduced also ATMS1981* and IRF5 upregulation, in comparison to control cells. These results demonstrate that the induction of NOX2-dependent ATM activation is required for tuning macrophages toward a pro-inflammatory phenotype.
The alteration of NOX2-dependent tumor macrophage activation is associated with poor prognosis after radiotherapy. Despite the fact that neoadjuvant chemoradiotherapy for locally advanced rectal cancer patients improve the local control of the tumors, only $15 \%$ of patients exhibit a complete response to treatment. ${ }^{37}$ In this context, we determined whether the perturbation of the signaling pathway that we involved in the macrophage activation toward a pro-inflammatory phenotype might be associated with the absence of local response to radiotherapy. We analyzed resected specimens of rectal cancer patients obtained after neoadjuvant radiotherapy performed before radical tumor resection. According to the tumor regression grade (TRG) criteria, these patients were classified into 'good responders' (TRG $\leqslant 2, n=29$ ) and 'bad responders' (TRG $\geqslant$ $3, n=27$ ) (Supplementary Table 1). We analyzed the total number of $\mathrm{CD}^{+} 8^{+}$TAM in both groups of irradiated tumors, and did not detect a significant difference in the CD68 ${ }^{+}$TAMs infiltration (Figure 6a). In addition, we also detected the autophosphorylation ATMS1981* in $\sim 20 \%$ of TAMs (Figure $6 \mathrm{~b}$ ), but we did not observe a significant difference for the frequencies of TAMs exhibiting ATMS1981* (ATMS1981*+ $\mathrm{CD}^{+} \mathrm{8}^{+}$) between the two groups of tumors (Figure 6c). Interestingly, we detected a significant increase in the frequency of TAMs revealing an enhanced expression of iNOS (iNOS ${ }^{+} \mathrm{CD}^{+} 8^{+}$) in tumor samples obtained from 'good responders' as compared to those obtained from 'bad responders' (Figures $6 \mathrm{~d}$ and e), confirming that macrophage activation toward a pro-inflammatory phenotype is associated with the local tumor control. Finally, we found a higher frequency of TAMs showing an upregulation of 

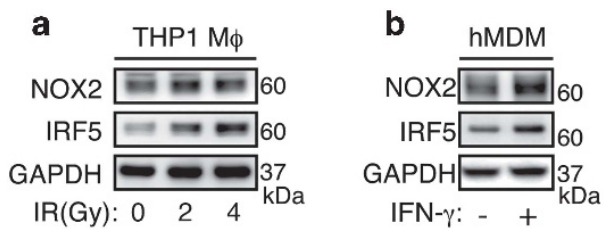

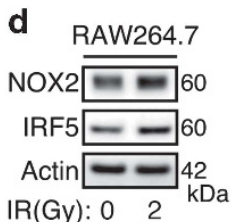

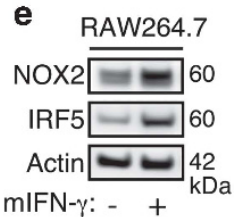

f

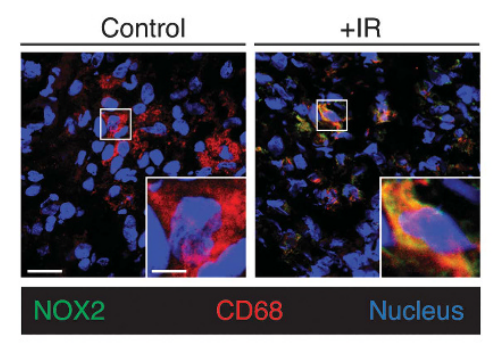

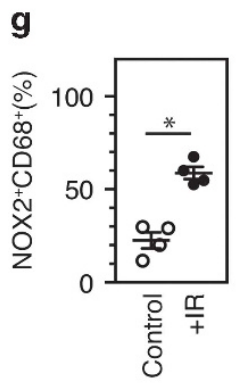

h

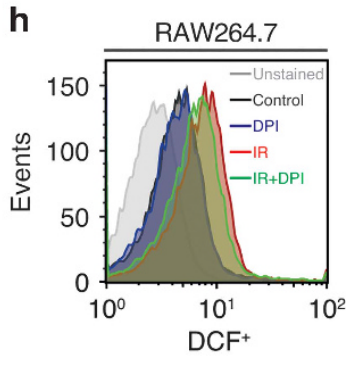

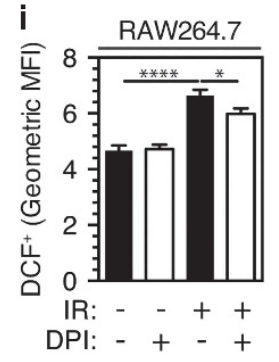
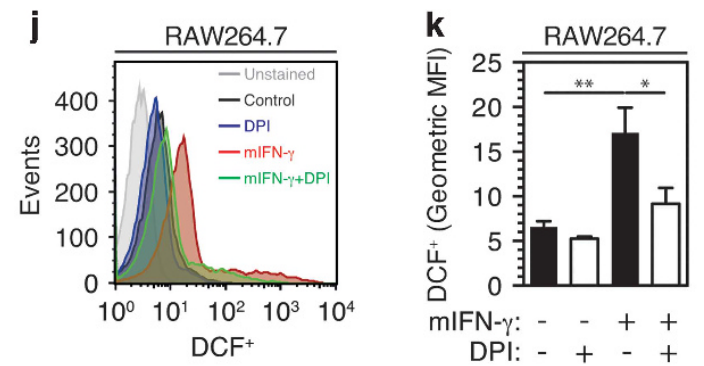

I

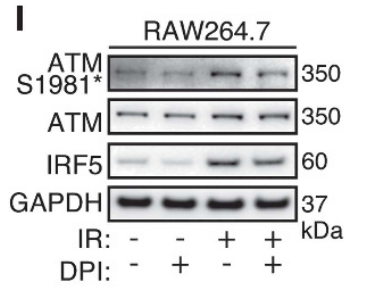

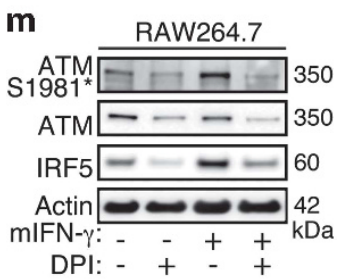

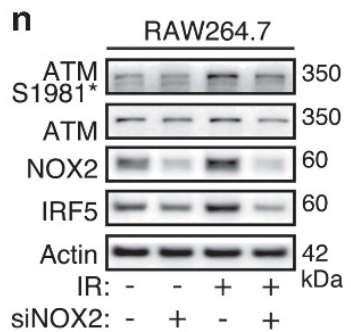

Figure 5 NOX2-dependent ROS production is involved in the pro-inflammatory macrophage activation. (a-e) NOX2 and IRF5 expressions after, respectively, 96 and $6 \mathrm{~h}$ culture of PMA-differentiated human THP1 macrophages (a) or murine RAW264.7 macrophages (d) that have been irradiated (or not) with indicated doses (a and $\mathbf{d}$ ); or $24 \mathrm{~h}$ culture of hMDM with $4 \mu \mathrm{g} / \mathrm{ml}$ of human IFN- $\gamma(\mathbf{b})$, PMA-differentiated human THP1 macrophages with $20 \mathrm{ng} / \mathrm{ml}$ of human IFN- $\gamma$ (c) or murine RAW264.7 macrophages with $20 \mathrm{ng} / \mathrm{ml}$ of $\mathrm{mIFN}-\gamma$ (e) were determined. Representative immunoblots are shown. GAPDH and actin were used as loading control. (f, $\mathbf{g}$ ) Representative confocal micrographs and frequencies of NOX2 ${ }^{+} \mathrm{CD} 68^{+}$tumor-associated macrophages detected in absence or after 45 Gy total dose of fractionated irradiation on tumor samples obtained from locally advanced rectal cancer patients are shown (scale bar, $20 \mu \mathrm{m}$; scale bar of insert, $5 \mu \mathrm{m}$ ). Results are expressed as mean value \pm S.E.M. $P$-value $\left({ }^{*} P<0.05\right)$ was calculated using Mann-Whitney U-test. (h-k) Murine RAW264.7 macrophages treated with $200 \mathrm{~nm}$ of DPI and irradiated with 2 Gy single dose (h, i) or stimulated with $20 \mathrm{ng} / \mathrm{ml} \mathrm{mIFN}-\gamma(\mathbf{j}, \mathbf{k})$ stained with $\mathrm{H}_{2} \mathrm{DCFDA}$ and analyzed by flow cytometry. Representative flow cytometry analysis and quantifications of geometric MFI are shown. Data are presented as means \pm S.E.M. in $\mathbf{i}$ and $\mathbf{k}$ panels. Significances are ${ }^{*} P<0.05,{ }^{* \star} P<0.01$ and ${ }^{* * * *} P<0.0001$, and were obtained using one-way ANOVA test. (I-o) ATMS1981 ${ }^{*}$, ATM and IRF5 expressions after, respectively, 6 and $24 \mathrm{~h}$ culture of murine RAW264.7 macrophages that have been incubated with $200 \mathrm{~nm}$ of DPI (I, m) or depleted for NOX2 (n, o) and irradiated with 2 Gy single dose $(\mathbf{l}, \mathbf{n})$ or treated with $20 \mathrm{ng} / \mathrm{ml} \mathrm{mIFN}-\gamma(\mathbf{m}, \mathbf{0})$ were determined. Representative immunoblots are shown. GAPDH and actin were used as loading control. Quantification of western blot bands are shown in Supplementary Figure 6

NOX2 expression $\left(\mathrm{NOX}_{2}^{+} \mathrm{CD} 68^{+}\right)$in resected specimens obtained from 'good responders', as compared to those obtained from 'bad responders' (Figures $6 f$ and g), revealing that the detection of NOX2 expression on TAMs may serve as a predictive factor for radiotherapy effectiveness. Multivariate statistical analysis confirmed these results (Table 1). Altogether, these results confirm that the NOX2 $\rightarrow$ ROS $\rightarrow$ ATMS $1981^{*}$ cascade may contribute to an efficient macrophage activation in response to radiotherapy.

\section{Discussion}

Phenotypic and functional plasticity are key features of immune cells. ${ }^{38}$ TAMs display a mixed functional phenotype with a majority of alternative features ${ }^{39}$ that have been associated with tumor growth and resistance to anticancer therapies. Although reprogramming TAMs represents a promising approach to enhance cancer therapies, ${ }^{40-44}$ molecular mechanisms underlying IRelicited macrophage activation have been poorly characterized. 
a

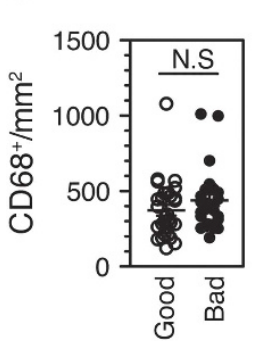

e

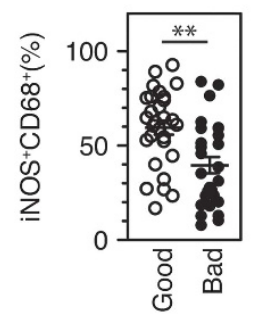

b

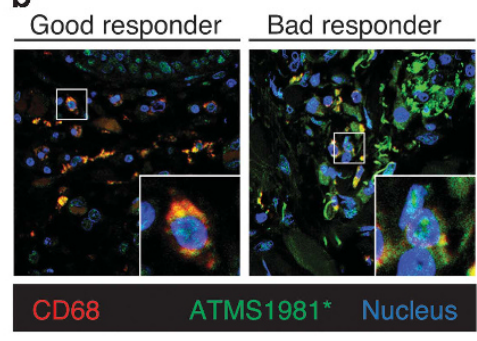

f

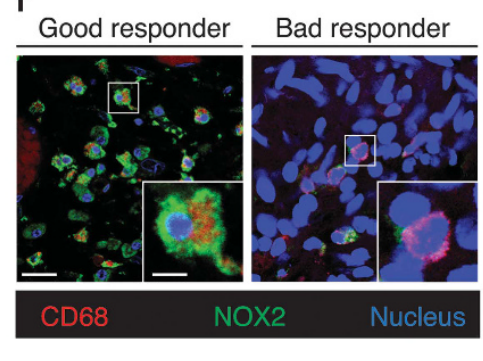

c

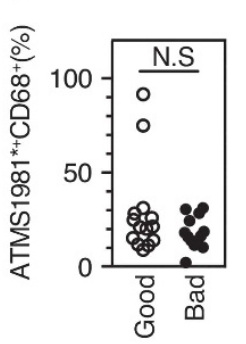

d

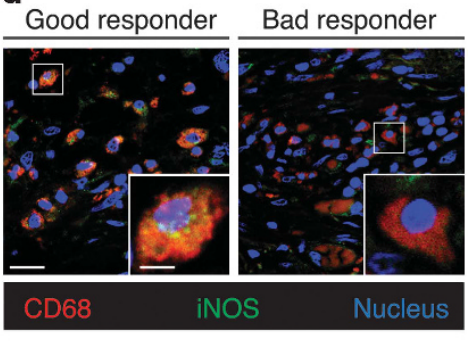

g

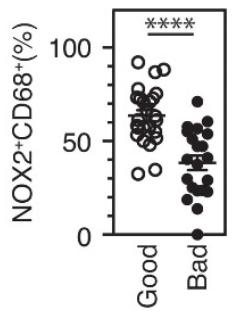

Figure 6 The perturbation of NOX2/ATM-dependent signaling pathway is associated with poor tumor response to radiation therapy.(a) Densities of CD68 ${ }^{+}$tumor-infiltrating macrophages detected on biopsies of human rectal tumor samples from good responders $(n=29)$ and bad responders $(n=27)$ to neoadjuvant radiation therapy were analyzed. Data are presented as means \pm S.E.M. (b) Representative confocal micrographs and frequencies of ATMS1981 ${ }^{*+} \mathrm{CD}_{68}{ }^{+}(\mathbf{b}, \mathbf{c})$, iNOS ${ }^{+} \mathrm{CD} 68^{+}(\mathbf{d}, \mathbf{e})$ or NOX2 ${ }^{+} \mathrm{CD} 68^{+}(\mathbf{f}, \mathbf{g})$ tumorassociated macrophages detected in good responders $(n=29)$ and bad responders $(n=27)$ to neoadjuvant radiation therapy are shown (scale bar, $20 \mu \mathrm{m})$. Representative

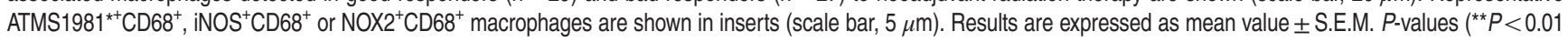
and ${ }^{* \star *} P<0.001$ ) were calculated using Mann-Whitney U-test

Table 1 Multivariate analysis of macrophage histological markers in rectal cancer response to neoadjuvant radiotherapy

\begin{tabular}{|c|c|c|c|}
\hline & $\begin{array}{l}\text { OR TRG (3-4-5) } \\
\text { versus }(1-2)\end{array}$ & $95 \% \mathrm{Cl}$ & $P$-value \\
\hline $\begin{array}{l}C D 68^{+} / \mathrm{mm}^{2} \\
\text { Median cutoff } \\
<376.23 \\
\geqslant 376.23\end{array}$ & $\begin{array}{c}1 \\
1.369\end{array}$ & $(0.374-5.010)$ & 0.635 \\
\hline $\begin{array}{l}\text { iNOS }^{+} / \mathrm{CD}^{+} 8^{+}(\% \\
\text { Median cutoff } \\
\quad<53.72 \\
\geqslant 53.72\end{array}$ & $\begin{array}{c}1 \\
0.089\end{array}$ & $(0.018-0.431)$ & 0.003 \\
\hline $\begin{array}{l}N O X 2^{+} / C D 68^{+} \\
\text {Median cutoff } \\
<55.07 \\
\geqslant 55.07\end{array}$ & $\begin{array}{c}1 \\
0.077\end{array}$ & $(0.013-0.472)$ & 0.006 \\
\hline $\begin{array}{l}\text { ATMS1981*+ } / C D \\
\text { Median cutoff } \\
<14.93 \\
\geqslant 14.93\end{array}$ & $\begin{array}{c}1 \\
0.513\end{array}$ & $(0.131-2.013)$ & 0.339 \\
\hline
\end{tabular}

Abbreviations: $\mathrm{Cl}$, confidence interval; OR, odds ratios.

The statistical comparisons of indicated histological markers between 'good responders' (TRG $\leqslant 2, n=29$ ) and 'bad responders' (TRG $\geqslant 3, n=27$ ) have been adjusted on TNM stages, time interval between radiotherapy and surgery, and concomitance with chemotherapy. Median cutoff, OR and $95 \% \mathrm{Cl}$ are indicated. $P$-values were calculated using Wald test and significant $P$ values are indicated in bold

In this paper, we showed that the in vitro treatment of macrophages with various doses of IR led to their activation toward a pro-inflammatory phenotype. Our observations were confirmed using xenograft tumor models and human rectal cancer specimens obtained from patients that have been treated with chemoradiotherapy. Consistent with other in vitro and in vivo studies, ${ }^{13,15,45,46}$ our results revealed that the transcription factor IRF5, which is the major regulator of proinflammatory macrophage phenotype, ${ }^{15}$ is activated in response to IR (as revealed by the upregulated expressions of IRF5- and IRF5-dependent target genes (such as IL-6, TNF$a$ or IFN- - )). IRF5, which is involved in the regulation of the expression of pro-inflammatory cytokine genes and in the repression of anti-inflammatory cytokine genes (such as IL-10), ${ }^{15,47}$ plays also a central role in hematopoietic cell development ${ }^{48}$ and in the susceptibility to inflammatory autoimmune diseases (such as systematic lupus erythematosus, rheumatoid arthritis and multiple sclerosis), ${ }^{49-51}$ demonstrating that the expression of IRF5 is tightly regulated during macrophage homeostasis.

Considering that IR mainly acts through the induction of DNA damages and the production of ROS, ${ }^{52,53}$ we then investigated the role of DNA damage response and ROS production during this process. In this paper, we initially reported that the DNA damage/repair kinase ATM is activated by IR and by classical macrophage activators (such as LPS and IFN- $\gamma$ ), and that DNA damage/repair ATM kinase is required for macrophage activation. Despite the fact that the ATM kinase may contribute to the development of T cells ${ }^{54}$ and of professional antigen-presenting cells (such as macrophage and dendritic cells) ${ }^{55,56}$ and modulate functions of immune cells (such as STING-dependent macrophage production of type I IFNs ${ }^{57}$ ), the contribution of ATM to macrophage activation was never investigated. Here, we found that IR induced the phosphorylation of ATM (on serine 1981) and of 
the histone $\mathrm{H} 2 \mathrm{AX}$ (on serine 139) in a time- and dosedependent manner in macrophages, indicating that DNA double-strand breaks and DNA damage response are elicited during macrophage activation. Moreover, our results also demonstrated that ATM inhibition reduces IRF5 mRNA level, highlighting that the biological activity of the kinase ATM controls macrophage activation through the regulation of IRF5 transcription. Despite the fact that several molecular components of DNA damage response pathways (such as Nijmegen breakage syndrome 1 (NBS1) protein ${ }^{58}$ ) have already been involved in monocyte/macrophage development and functions, the cellular events and signaling pathways that lead to ATM activation and control IRF5 expression during macrophage activation were until now never characterized.

In this study, we also identified the NOX2-dependent ROS production as an upstream second messenger required for ATM phosphorylation and macrophage activation. We found that the expression of NOX2 was increased after IR or IFN- $\gamma$ treatment. The increased NOX2 expression that we detected both in vitro and in 'good responders' to radiotherapy might be regulated at transcriptional level (through the activation of PU.1 or NF- $k$ B transcription factors ${ }^{35}$ ) or at post-transcriptional level. ${ }^{35}$ Increased expression of NOX2 then led to the generation of ROS that contributes to double-strand breaks formation, induces the activation of ATM and IRF5, and favors the functional switch of macrophages from anti-inflammatory to pro-inflammatory phenotype (Figure 7). Although we demonstrated that NOX2 expression controlled macrophage activation after IR or IFN- $\gamma$ stimulation, the precise mechanisms involved in NOX2 expression or linking DNA doublestand breaks formation, phosphorylation of ATM (ATMS1981*) and IRF5 expression are under active investigations. In this study, we also demonstrated that the histological detection of any components of the molecular cascade that we described in macrophages (NOX2 $\rightarrow$ ROS $\rightarrow$ ATMS1981* $\rightarrow$ IRF5) predicted the effectiveness of radiotherapy and might also help for the prediction of other anticancer treatments.

We also showed that the modulation of these components impacts the reprogramming of macrophages elicited by IR or IFN- $\gamma$. We found that ROS scavenging or inhibition of NOX2 or ATM activity interrupts this molecular cascade in vitro. Moreover, we did not detect the induction of this signaling pathway on tumor samples obtained from 'bad responders' as compared to those obtained from 'good responders'. More importantly, we demonstrated that modulators of DNA repair (such as Olaparib) that are currently evaluated in combination with radiotherapy triggered this signaling pathway and resulted in macrophage activation toward a pro-inflammatory phenotype. In this context, the stimulation of NOX2 activity, the enhanced generation of ROS, the inhibition of DNA repair, the activation of ATM or the transactivation of IRF5 could constitute effective strategies to enhance radiotherapy efficacy in clinic. Therefore, our results suggested that macrophage activation toward NOX2-/ATM-dependent proinflammatory phenotype was involved in the tumor response to chemoradiotherapy (with no impact on the overall survival and disease-free survival of patients). We propose that by combining radiotherapy with other modalities of cancer treatments (such as PARP inhibitors) might enhance the

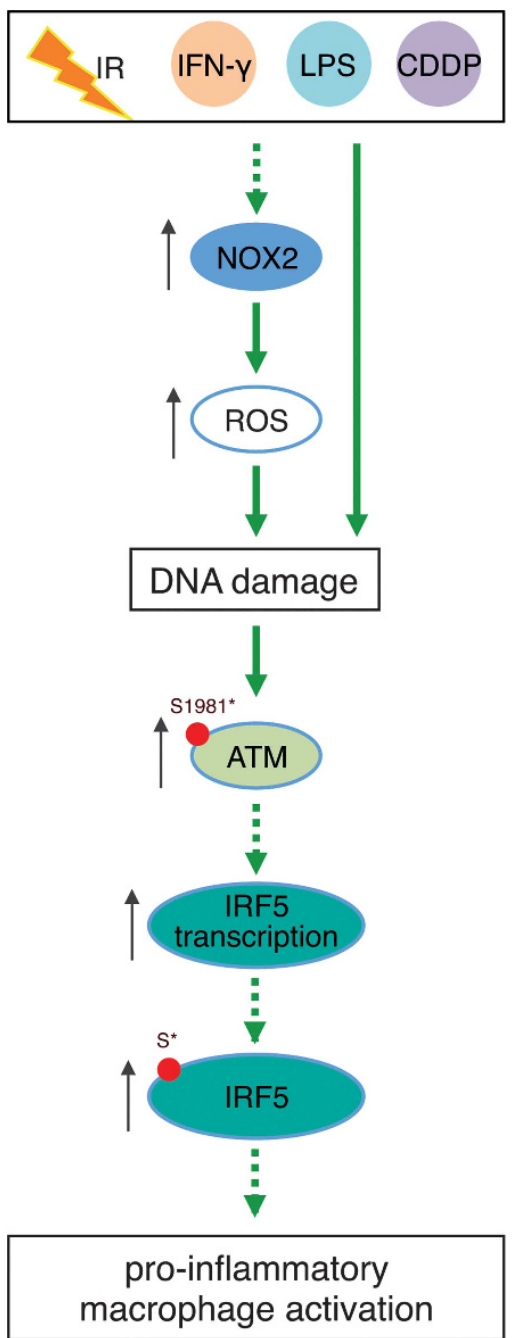

Figure 7 Proposed model for the roles of NOX2 and ATM activations in proinflammatory macrophage activation

tumor response to radiotherapy and lead to a long-term benefit to rectal patients.

Materials and Methods

Cells and reagents. The human monocyte cell line THP1 cells and the murine macrophage-like RAW264.7 cells were maintained in RMPI-1640-Glutamax medium (Life Technologies, Carlsbad, CA, USA) supplemented with 10\% heat-inactivated fetal bovine serum (FBS) (Hycultec GmbH, Beutelsbach, Germany) and $100 \mathrm{IU} / \mathrm{ml}$ penicillin-streptomycin (Life Technologies). To obtain THP1 macrophages, THP1 monocytes were differentiated with $320 \mathrm{nM}$ of PMA (\#tlrl-PMA, Invivogen, San Diego, CA, USA) during $24 \mathrm{~h}$. Then, cells were washed three times to remove PMA and non-adherent cells. For the generation of hMDM, buffy coats from healthy donor blood were obtained from the French blood bank (Etablissement Français du Sang) under the control of convention with the INSERM. In accordance with French law, written informed consent for the use of cells for clinical research was obtained from each donor. Monocytes were obtained from buffy coats and were differentiated into macrophages by using human $A B$ serum in macrophage medium, as previously described. ${ }^{59}$ After 7-day differentiation, hMDM were harvested and suspended in macrophage medium containing $10 \%$ (vol/vol) heat-inactivated FBS, yielding from 91 to $96 \%$ of CD14-positive cells that expressed macrophage differentiation markers (C11b and CD71), and macrophage alternative activation markers (CD163). All cells were maintained under $5 \% \mathrm{CO}_{2}$ humidified atmosphere at $37^{\circ} \mathrm{C}$. Dimethyl sulfoxide 
(DMSO, \#D2650), LPS (\#L2880), N-acetyl-L-cysteine (NAC, \#A7250), diphenyleneiodonium chloride (DPI, D2926), NCZ (\#N9162), cis-diammineplatinum(II) dichloride (cisplatin) (CDDP, \#P4394) were purchased from Sigma-Aldrich (St. Louis, MO, USA). Recombinant murine IFN- $\gamma$ (mIFN- $\gamma$, $\sharp 315-05)$ was obtained from PeproTech (Neuilly-sur-seine, France). Recombinant Human IFN- $\gamma$ (IFN- $\gamma, \# 285-$ IF/CF) was from R\&D Systems (Minneapolis, MN, USA). KU55933 (\#3544/10) was from Tocris Bioscience (Bristol, UK). Mn(III)tetrakis (Fontenay-sousbois, France) (4-benzoic acid) porphyrin chloride (MnTBAP, \#475870) was from Calbiochem (San Diego, CA, USA). Olaparib (\#S1060) was from Selleckchem (Houston, TX, USA). PMA (\#trl-PMA) was from Invivogen.

Antibodies. Antibodies used for immunofluorescence were anti-phospho-ATM (Ser1981) (\#ab36810), anti-iNOS (\#3523) antibodies from Abcam (Cambridge, UK), anti-53BP1 (\#4937) antibody from Cell Signaling Technology (Danvers, MA, USA), anti-phospho-H2AX (Ser139) (\#05-636) antibody from EMD Millipore (Billerica, MA, USA). Antibodies used for immunoblots were anti-phospho-ATM (Ser1981) (10H11. E12) (\#4526) and anti-ATM (D2E2) (\#2873) antibodies from Cell Signaling Technology; anti-IRF5 (\#ab21689), anti-phospho-serine (\#ab9332) and anti-IL-1/ (\#ab2105) antibodies were from Abcam; anti-gp91-phox (54.1) (NOX2) (\#sc-13054) antibody was from Santa Cruz (Dallas, TX, USA). Anti-GAPDH antibody (\#MAB374, EMD Millipore) or anti-beta actin antibody (AC-15) (HRP) (\#49900, Abcam) was used as a loading control. Phenotypic analyses on primary human MDMs were realized by flow cytometry using anti-CD14 (PE) (\#12-1049-42, eBioscience, Illkrich, France), anti-CD11b (APC-Cy7) (\#557657, BD Pharmingen, Le pont de Claix, France), anti-CD71 (PE) (\#555537, BD Pharmingen, Le pont de Claix, France) and anti-CD163 (Alexa Fluor 647) (\#562669, BD Pharmingen) antibodies. For immunohistochemistry staining, anti-mouse CD11b (Clone M1/70, \#550282) antibody was purchased from BD Biosciences (Le pont de Claix, France); antiphospho-ATM (Ser1981) (EP1890Y, \#GTX61739) antibody was from GeneTex (Irvine, CA, USA); anti-gp91-phox (54.1) (NOX2) antibody was from Santa Cruz (sc130534, Dallas, TX, USA); anti-phospho-H2AX (Ser139) (\#05-636) was from EMD Millipore, anti-iNOS (\#ab3523) was from Abcam and anti-human CD68 antibodies were, respectively, from DAKO (Santa Clara, CA, USA) (\#MO876) and Thermo Fischer Scientific (\#PA5-32331, Illkrich, France).

Macrophage activation. Human MDM $\left(10^{6}\right)$ were activated by treatment with $4 \mu \mathrm{g}$ recombinant human IFN- $\gamma$ for $24 \mathrm{~h}$. THP1 monocytes were differentiated into macrophages by $320 \mathrm{~nm}$ PMA for $24 \mathrm{~h}$. Then, macrophages were activated with $20 \mathrm{ng} / \mathrm{ml}$ recombinant human IFN- $\gamma$ or $100 \mathrm{ng} / \mathrm{ml}$ LPS during $24 \mathrm{~h}$. RAW264.7 macrophages were activated with $20 \mathrm{ng} / \mathrm{ml}$ recombinant murine IFN- $\gamma$ or $100 \mathrm{ng} / \mathrm{ml}$ LPS for $24 \mathrm{~h}$.

Irradiation. Cells were seeded in 6-well plates, 12-well plates or $25 \mathrm{~cm}^{2}$ flasks and irradiated with gamma-ray irradiator $\mathrm{IBL}-637\left(\mathrm{Cs}^{137}, 1 \mathrm{~Gy} / \mathrm{min}\right.$, gamma CIS-Biolnternational, IBA, Saclay, France) or with X-ray irradiator (1 Gy/min, X-RAD 320, Precision X-Ray). Cells were harvested at indicated time points (hMDMs and THP1 macrophages at $96 \mathrm{~h}$, RAW264.7 macrophages at $6 \mathrm{~h}$ for cell lysates and $12 \mathrm{~h}$ for supernatants) after irradiation for subsequent experiments.

RNA-mediated interference. The SMARTpool siGENOME ATM siRNA (M-003201-04-0005) against ATM (siRNA ATM), SMARTpool siGENOME CYBB siRNA (M-011021-01-0005) against NOX2 (siRNA NOX2) and siGENOME NonTargeting siRNA Pool \#1 (D-001206-13-05) as control were purchased from Dharmacon (Lafayette, CO, USA). siRNA-5 control, siRNA-4 and siRNA-5 against ATM were from Sigma-Aldrich. Sequences of siRNAs are as follows: SMARTpool siGENOME CYBB siRNAs (containing siRNA-1: $5^{\prime}$-GAAGACAACUGGACAGGAA-3'; siRNA-2: 5'-GGAACUGGGCUGUGAAUGA-3'; siRNA-3: 5'-GUGAAUGCCCGAGU CAAUA-3' and siRNA-4: 5'-GAAACUACCUAAGAUAGCG-3'); SMARTpool siGENOME ATM siRNAs (containing siRNA-1: 5'-GCAAAGCCCUAGUAACAUA-3'; siRNA-2: 5'-GGGCAUUACGGGUGUUGAA-3'; siRNA-3: 5'-UCGCUUAGCAGGA GGUGUA-3'; siRNA-4: 5'-UGAUGAAGAGAGACGGAAU-3'); ATM siRNA-5 (5'-UG AAGUCCAUUGCUAAUCA-3'); ATM SiRNA-6 (5'-AACAUACUACUCAAAGACA-3') and control siRNA-5 (5'-UUCAAUAAAUUCUUGAGGU-3'). Sequences of ATM siRNA-5, ATM siRNA-6 and SMARTpool siGENOME CYBB siRNA (M-011021-01. 0005) that we used are, respectively, perfectly aligned against mouse ATM or NOX2 genes. The control siGENOME Non-Targeting siRNAs were a pool of four on-target plus non-targeting siRNAs. INTERFERin Reagent (\#409-10, Polyplus Transfection, Illkrich, France) was used as the siRNA transfection reagent for hMDM and PMA-differentiated THP1 macrophages according to the manufacturer's instructions.
Transfection of hMDM was performed as previously described. ${ }^{59}$ Briefly, hMDM were seeded $\left(2.5 \times 10^{5} \mathrm{hMDM} / 0.25 \mathrm{ml}\right.$ per well in 24-well plate in macrophages medium $+10 \% \mathrm{FBS}$ ) and were allowed to adhere to the substrate by culturing at $37^{\circ}$ $\mathrm{C}$ for $2 \mathrm{~h}$ prior to siRNAs transfection. siRNAs were pre-diluted in $125 \mu \mathrm{l}$ of OptiMEM (Thermo Fisher Scientific) in which $10 \mu$ of INTERFERin were then added. The transfection mix was left to incubate at room temperature for $15 \mathrm{~min}$ and was added to hMDM to achieve the final concentration of $100 \mathrm{~nm}$ siRNAs. The MDMs were then incubated at $37^{\circ} \mathrm{C}$ for $24 \mathrm{~h}$. The medium was replaced by fresh macrophage medium supplemented with $10 \%$ FBS before subsequent experiments. Lipofectamine RNAi max (\#13778150, Life Technologies, Illkrich, France) was used to transfect RAW264.7 macrophages according to the manufacturer's instructions. Briefly, RAW264.7 cells were seeded ( $10^{5}$ cells per $\mathrm{ml}$ per well in 12-well plate) and were allowed to adhere to the substrate by culturing at $37^{\circ} \mathrm{C}$ for $24 \mathrm{~h}$ prior to siRNAs transfection. The transfection mix was added to the final concentration of $10 \mathrm{~nm}$ siRNAs. The RAW264.7 cells were then incubated at $37^{\circ} \mathrm{C}$ for $24 \mathrm{~h}$ before subsequent experiments.

Immunofluorescence microscopy and flow cytometry. Cells were grown on coverslips and were treated as indicated. After treatment, cells were rinsed twice, fixed with 10\% neutral buffered formalin (Sigma-Aldrich) for $10 \mathrm{~min}$ and then permeabilized with $0.3 \%$ Triton X-100 in PBS for $15 \mathrm{~min}$. Cells were then washed twice with PBS and were blocked with $10 \%$ FBS in PBS for $1 \mathrm{~h}$ at room temperature, followed by incubation with primary antibodies in $10 \%$ FBS in PBS for $1 \mathrm{~h}$ at $30^{\circ} \mathrm{C}$ at room temperature. Then, samples were incubated with secondary antibodies using Alexa Fluor 488 green or Alexa Fluor-546 red (Life Technologies, Illkrich, France) and Hoechst 33342 for nuclei (Thermo Fisher Scientific) in 10\% FBS in PBS for $30 \mathrm{~min}$ at room temperature. Coverslips were mounted with Fluoromount-G (SouthernBiotech, Birmingham, AL, USA) and then visualized with Leica TCS SPE confocal microscope (Leica Microsystems, Nanterre, France) using a $\times 63$ objective. In experiments of 53BP1 and $\gamma-\mathrm{H} 2 \mathrm{AX}$ foci visualization in irradiated THP1 cells, $Z$ series of optical sections at $1 \mu \mathrm{m}$ increments was acquired. In experiments of ATMS $1981^{*}$ and $\gamma$-H2AX foci visualization in RAW264.7 cells treated with $\mathrm{mIFN}-\gamma$, LPS or CDDP, $Z$ series of optical sections at $1.7 \mu \mathrm{m}$ increments were acquired. In experiments of ATMS1981* and $\gamma$-H2AX foci visualization in RAW264.7 cells treated with KU55933 and irradiated, Z series of optical sections at $2.0 \mu \mathrm{m}$ increments were acquired. For flow cytometry analysis, PMA-differentiated THP1 macrophages were fixed in $4 \%$ paraformaldehyde-PBS for $15 \mathrm{~min}$ and permeabilized with $0.03 \%$ Triton $X-100$ (Sigma-Aldrich) for $15 \mathrm{~min}$ at room temperature. Cells were then blocked with FBS during $1 \mathrm{~h}$ at $4{ }^{\circ} \mathrm{C}$ and incubated as indicated for $30 \mathrm{~min}$ at $4^{\circ} \mathrm{C}$, with anti-phospho-ATM (Ser1981) (EP1890Y) (\#GTX61739, GeneTex, Irvine, CA, USA), anti-phospho-H2AX (Ser139) (\#05-636, EMD Millipore) or anti-iNOS (\#ab3523, Abcam) antibodies. Then, cells were incubated with the secondary antibodies conjugated to Alexa Fluor 488 fluorochrome (Life Technologies) for $30 \mathrm{~min}$ at $4{ }^{\circ} \mathrm{C}$ and analyzed with Guava flow cytometer (EMD Millipore).

Immunoprecipitation and western blots. Cells were washed twice with cold PBS and lysed with NEHN buffer (0.5\% NP40, 20\% Glycerol, $300 \mathrm{~mm} \mathrm{NaCl,}$ $20 \mathrm{~mm}$ Hepes, pH 7.5 and $1 \mathrm{~mm}$ EDTA) complemented with $2.5 \mathrm{~mm}$ DTT, and the protease and phosphatase inhibitor (Roche, Basel, Switzerland) at $4{ }^{\circ} \mathrm{C}$. About $5-$ $20 \mu \mathrm{g}$ of proteins were separated by NuPAGE $4-12 \%$ or $10 \%$ SDS-PAGE gel (Invitrogen, Illkrich, France), and then were transferred onto a nitrocellulose membrane (0.2 $\mu$, Bio-Rad, Marnes-la-coquette, France). Membranes were blocked with $5 \%$ non-fat milk or $5 \%$ bovine serum albumin (BSA) in Tris-buffered saline and $0.1 \%$ Tween 20 (TBS-T) at room temperature for $1 \mathrm{~h}$ and then subsequently probed with primary antibodies overnight at $4^{\circ} \mathrm{C}$. Then, membranes were incubated with appropriate horseradish peroxidase-conjugated anti-rabbit or anti-mouse IgG (SouthernBiotech, Birmingham, AL, USA) for $1 \mathrm{~h}$ at room temperature. After three washes with TBS-T, immunoblots were revealed using G:BOX Chemi XL1.4 Fluorescent \& Chemiluminescent Imaging System (Syngene, Cambridge, UK). For immunoprecipitations, 2 Gy-irradiated RAW264.7 cells were harvested and lysed in NEHN buffer. About $2 \mathrm{mg}$ of total lysates were incubated with $2 \mu \mathrm{g}$ of anti-IRF5 antibodies (\#ab21689, Abcam) or with anti-phospho-serine antibodies (\#ab9332, Abcam) at $4{ }^{\circ} \mathrm{C}$ on the wheel for overnight. Then, the immunocomplexes were precipitated with protein $\mathrm{G}$ immobilized on sepharose beads for further $4 \mathrm{~h}$ at $4{ }^{\circ} \mathrm{C}$. After three washings in NEHN buffer containing $300 \mathrm{~mm} \mathrm{NaCl}$ and one in NEHN buffer with $600 \mathrm{~mm} \mathrm{NaCl}$, immunoprecipitates and protein inputs were boiled in the Laemmli buffer (Bio-Rad, Marnes-la-coquette, France) for $10 \mathrm{~min}$ at $95^{\circ} \mathrm{C}$ and analyzed by western blot for the indicated antibodies. 
Detection of ROS production. Hydrogen peroxide and anion superoxide production were determined by staining cells with $5 \mu \mathrm{M}$ of 2,7-dichlorodihydrofluorescein diacetate $\left(\mathrm{H}_{2} \mathrm{DCFDA}\right.$, \#D6883, Sigma-Aldrich) for $40 \mathrm{~min}$ at $37^{\circ} \mathrm{C}$. Cells were then washed twice with Hanks' balanced salt solution (HBSS, Thermo Fisher Scientific, Illkrich, France) and suspended in cold HBSS solution containing 1\% FBS for FACS analysis.

Determination of LDH release. The release of LDH in the supernatants of cultured cells was detected using Cytotoxicity Detection KitPLUS (LDH, \#04744926001) from Roche according to the manufacturer's instructions.

Quantitative RT-PCR. Total RNA of 2 Gy-irradiated PMA-differentiated THP1 macrophages and control cells were purified using RNeasy kit (Qiagen, Hilden, Germany) after $96 \mathrm{~h}$ post irradiation according to the manufacturer's instructions. For the detection of cytokine mRNA, the synthesis of CDNA and the quantitative PCR procedure using Syber Green were performed as previously described. ${ }^{60}$ The sequences of the primers used are the following: $\left(5^{\prime}-3^{\prime}\right)$ IL-23 (F:GTTCTGCTT GCAAAGGATCCA, R:TATCCGATCCTAGCAGCTTCTCA), IL-6 (F:GCTGCAGGCAC AGAACCA, R:ACTCCTTAAAGCTGCGCAGAA), TNF $\alpha$ (F:GGAGAAGGGTGACCG ACTCA, R:TGCCCAGACTCGGCAAAG), IFN $\gamma$ (F:AACTCATCCAAGTGATGGCTG AA, R:CTGACTCCTTTTTCGCTTCCCTG) and HPRT1 (F:GGACAGGACTGAACG TCTTGC, R:CTTGAGCACACAGAGGGCTACA). All samples were normalized with the endogenous hypoxanthine phosphoibosyltransferase 1 (HRPT1) mRNA. For the detection of IRF5 and ATM mRNA, the cDNA synthesis and TaqMan qPCR procedures were performed as previously described. ${ }^{59}$ The used probes of IRF5 (Hs $00158114 \mathrm{~m} 1)$, ATM (Hs01112355 gl) and GAPDH (Hs02758991 gl) were included in the premade TaqMan Gene expression mixes obtained from Applied Biosystems (Foster City, CA, USA). Results were analyzed with the cycle threshold methods $\left(C_{T}\right)$ and each sample was normalized to the quantity of endogenous GAPDH mRNA.

Human cytokine profiling. Human MDMs were irradiated at 4 Gy and were further incubated for $96 \mathrm{~h}$. The supernatants were harvested, centrifuged and stored at $-80^{\circ} \mathrm{C}$ until use. Human cytokines in these supernatants were measured using the proteome profiler Human cytokine array panel A (proteome profiler, \#ARY005, R\&D Systems) according to the manufacturer's instructions. Briefly, membranes were blocked with the blocking buffer at room temperature for $1 \mathrm{~h}$. Supernatants of hMDMs were mixed with a biotinylated detection antibody cocktail and then incubated with the membranes overnight at $4^{\circ} \mathrm{C}$. Membranes were washed three times for $10 \mathrm{~min}$ and subsequently incubated with streptavidin-horseradish peroxidase for $30 \mathrm{~min}$ at room temperature. Membranes were then washed three times for 10 min and exposed to peroxidase substrate, and revealed with the G:BOX Chemi XL1.4 Fluorescent and Chemiluminescent Imaging System (Syngene). Time of exposure was between 1 and $10 \mathrm{~min}$. The images were then analyzed using GeneTools software gel image analysis (Syngene).

In vivo mouse tumor model. To generate xenograft tumor model, $4 \times 10^{6}$ human colorectal HCT116 cells were inoculated subcutaneously in the flanks of 5week female nude mice. Two weeks later, the tumors were irradiated at 20 Gy using Variant-NDI-226-n^87262-YO X-Ray Tube. Tumor volume was monitored every 4-5 days. Mice were killed when tumors in the control group exceeded $1000 \mathrm{~mm}^{3}$.

Immunohistochemistry. Tumors obtained from in vivo experiments were resected, fixed and embedded in paraffin. Paraffin-embedded tumor samples from rectal patients undergoing neoadjuvant radiotherapy were kindly offered by Dr. Celine Mirjolet in Centre Georges-François Leclerc, Dijon. Frozen tumor samples from rectal patients before and after radiotherapy were obtained from Gustave Roussy Cancer Center. Tumor sections were then dried, deparaffinized and hydrated, followed by antigen retrieval with $0.01 \mathrm{M}$ sodium citrate buffer, $\mathrm{pH} 6.0$ at $97.6{ }^{\circ} \mathrm{C}$ for $20 \mathrm{~min}$. After washing with TBS-T, slides were blocked with $10 \% \mathrm{FBS}$ in PBS at room temperature for $1 \mathrm{~h}$. Then primary antibodies diluted in $10 \%$ FBS in PBS were applied to each section and incubated overnight in humidified chamber at $4{ }^{\circ} \mathrm{C}$. After three washes with TBS-T, Alexa Fluor-conjugated secondary antibodies and Hoechst 33342 diluted in 10\% FBS in PBS were applied to each section and incubated for $30 \mathrm{~min}$ at room temperature. Then, the slides were washed three times with TBS-T and once with water. Coverslips were mounted on slides using Fluoromount-G medium (SouthernBiotech) before visualization with Leica TCS SPE confocal microscope (Leica Microsystems) using a $\times 63$ objective.
Human samples. Human tissue samples of locally advanced rectal tumors that were resected 42 days after receiving $45 \mathrm{~Gy}(1.8 \mathrm{~Gy} / \mathrm{sessions})$ concomitantly to chemotherapy (5-FU) $(n=4)$ or left unirradiated $(n=4)$ were obtained from Gustave Roussy Cancer Campus (Villejuif, France). All tumor samples from responders and non-responders to chemoradiotherapy were obtained from Centre Georges François Leclerc (Dijon, France). This study was approved by the IRB and the French CCTIRS committee (Comité consultatif sur le traitement de l'information en matière de recherche et de santé) and CNIL (Commission nationale de l'informatique et des libertés). Characteristics of these patients are shown in Supplementary Table 1 . All these patients $(n=56)$ were diagnosed for locally advanced rectal tumors and characterized the Tumor Node Metastasis (TNM) classification. All human samples were obtained after approval by the institutional review board and ethics committee, with fully informed consents.

Statistical analysis. Two-way ANOVA test was used to establish statistical differences between the growth of non-irradiated and irradiated tumors in xenografted mice, Mann-Whitney U-test to establish statistical differences in biological activity (iNOS or NOX2 expression) and in phosphorylation $(\gamma-\mathrm{H} 2 \mathrm{AX}$ or ATMS1981*) of macrophages, and in the number of infiltrated CD11b cells between irradiated and non-irradiated tumors of xenografted mice or between tumors obtained from locally advanced rectal cancer patients who received or not 45 Gy total dose. Multivariate analysis with Wald test was used to determine statistical differences between tumors biopsies obtained from patients with locally advanced rectal tumors that respond or not to chemoradiotherapy. Statistical analysis of in vitro data was performed using Student's t-test or one-way ANOVA test (for Figures $2 p, 4 b, d, 5 i, k$ and Supplementary Figure 2j). Statistically significant values are reported in figure legends. All experiments were independently performed at least three times. Data are expressed as mean \pm S.E.M. GraphPad Prism version 6.0b (GraphPad Software, La Jolla, CA, USA) was employed to perform statistical analysis.

\section{Conflict of Interest}

The authors declare no conflict of interest.

Acknowledgements. This work was supported by funds from Agence Nationale de la Recherche (ANR-10-IBHU-0001, ANR-10-LABX33 and ANR-11IDEX-003-01), Electricité de France, Fondation Gustave Roussy, Institut National du Cancer (INCA 9414), NATIXIS, SIDACTION and the French National Agency for Research on AIDS and viral Hepatitis (ANRSH) (to J-LP.), Electricité de France and Fondation Gustave Roussy (to ED). QW is recipient of PhD fellowship of China Scholarship Council. AP and AA are, respectively, recipient of $\mathrm{PhD}$ fellowship and post-doc fellowship from Agence Nationale de Recherche sur le Sida et sur les Hépatites (ANRSH). LV and FL are recipient of PhD fellowships from Fondation pour la Recherche Médicale and CIFRE. HD, EM and MT are supported by the Laboratory of Excellence LERMIT with a grant from ANR (ANR-10-LABX-33) under the program 'Investissements d'Avenir' ANR-11-IDEX-0003-01. IM is funded by INCA (INCADGOS-INSERM 6043). CM work was supported by the 'Cancéropôle Grand Est', and the 'Conseils Régionaux de Bourgogne, de Franche Comté et de Lorraine'. We gratefully acknowledge S Solier, Y Lecluse and S Salome-Desnoulez for technical support.

\section{Author contributions}

QW, AA, AP, CL, CM, IM, LV, FL, HD, EM, ZM, MT, MG, OC and FM performed experiments. J-LP designed the study. QW, ED and J-LP analyzed the results, assembled the figures and wrote the paper. QW and CM performed statistical analysis. QW, DMO, FR, ES and NM provided advices and edited the paper.

1. Azzam El, Jay-Gerin JP, Pain D. lonizing radiation-induced metabolic oxidative stress and prolonged cell injury. Cancer Lett 2012; 327: 48-60.

2. Hekim N, Cetin Z, Nikitaki Z, Cort A, Saygili El. Radiation triggering immune response and inflammation. Cancer Lett 2015; 368: 156-163.

3. Park B, Yee C, Lee KM. The effect of radiation on the immune response to cancers. Int J Mol Sci 2014; 15: 927-943.

4. Kroemer G, Galluzzi L, Kepp O, Zitvogel L. Immunogenic cell death in cancer therapy. Annu Rev Immunol 2013; 31: 51-72. 
5. Ghiringhelli F, Apetoh L, Tesniere A, Aymeric L, Ma Y, Ortiz C et al. Activation of the NLRP3 inflammasome in dendritic cells induces IL-1beta-dependent adaptive immunity against tumors. Nat Med 2009; 15: 1170-1178.

6. Prakash H, Klug F, Nadella V, Mazumdar V, Schmitz-Winnenthal H, Umansky L. Low doses of gamma irradiation potentially modifies immunosuppressive tumor microenvironment by retuning tumor-associated macrophages: lesson from insulinoma. Carcinogenesis 2016; 37 301-313.

7. Merrick A, Errington F, Milward K, O'Donnell D, Harrington K, Bateman A et al. Immunosuppressive effects of radiation on human dendritic cells: reduced IL-12 production on activation and impairment of naive T-cell priming. Br J Cancer 2005; 92: 1450-1458.

8. Price JG, Idoyaga J, Salmon H, Hogstad B, Bigarella CL, Ghaffari S et al. CDKN1A regulates Langerhans cell survival and promotes Treg cell generation upon exposure to ionizing irradiation. Nat Immunol 2015; 16: 1060-1068.

9. Klug F, Prakash H, Huber PE, Seibel T, Bender N, Halama N et al. Low-dose irradiation programs macrophage differentiation to an iNOS(+)/M1 phenotype that orchestrates effective T cell immunotherapy. Cancer Cell 2013; 24: 589-602.

10. Chiang CS, Fu SY, Wang SC, Yu CF, Chen FH, Lin CM et al. Irradiation promotes an m2 macrophage phenotype in tumor hypoxia. Front Oncol 2012; 2: 89.

11. Franklin RA, Liao W, Sarkar A, Kim MV, Bivona MR, Liu K et al. The cellular and molecular origin of tumor-associated macrophages. Science 2014; 344: 921-925.

12. Mosser DM, Edwards JP. Exploring the full spectrum of macrophage activation. Nat Rev Immunol 2008; 8: 958-969.

13. Weiss M, Byrne AJ, Blazek K, Saliba DG, Pease JE, Perocheau D et al. IRF5 controls both acute and chronic inflammation. Proc Natl Acad Sci USA 2015; 112: 11001-11006.

14. Weiss M, Blazek K, Byrne AJ, Perocheau DP, Udalova IA. IRF5 is a specific marker of inflammatory macrophages in vivo. Mediators Inflamm 2013; 2013: 245804.

15. Krausgruber T, Blazek K, Smallie T, Alzabin S, Lockstone H, Sahgal N et al. IRF5 promotes inflammatory macrophage polarization and TH1-TH17 responses. Nat Immunol 2011; 12 231-238.

16. Satoh T, Takeuchi O, Vandenbon A, Yasuda K, Tanaka Y, Kumagai Y et al. The Jmjd3-Irf4 axis regulates $\mathrm{M} 2$ macrophage polarization and host responses against helminth infection. Nat Immunol 2010; 11: 936-944.

17. Cosin-Roger J, Ortiz-Masia D, Calatayud S, Hernandez C, Esplugues JV, Barrachina MD. The activation of Wnt signaling by a STAT6-dependent macrophage phenotype promotes mucosal repair in murine IBD. Mucosal Immunol 2016; 9: 986-998.

18. Kapoor N, Niu J, Saad Y, Kumar S, Sirakova T, Becerra E et al. Transcription factors STAT6 and KLF4 implement macrophage polarization via the dual catalytic powers of MCPIP. $J$ Immunol 2015; 194: 6011-6023.

19. Rodriguez-Zapata M, Reyes E, Sanchez L, Espinosa A, Solera J, Alvarez-Mon M. Defective reactive oxygen metabolite generation by macrophages from acute brucellosis patients. Infection 1997; 25: 187-188.

20. Colegio OR, Chu NQ, Szabo AL, Chu T, Rhebergen AM, Jairam V et al. Functional polarization of tumour-associated macrophages by tumour-derived lactic acid. Nature 2014 513: 559-563.

21. Wen Z, Liu H, Li M, Li B, Gao W, Shao Q et al. Increased metabolites of 5-lipoxygenase from hypoxic ovarian cancer cells promote tumor-associated macrophage infiltration. Oncogene 2015; 34: 1241-1252.

22. Mantovani A, Sozzani S, Locati M, Allavena P, Sica A. Macrophage polarization: tumorassociated macrophages as a paradigm for polarized M2 mononuclear phagocytes. Trends Immunol 2002; 23: 549-555.

23. Condeelis J, Pollard JW. Macrophages: obligate partners for tumor cell migration, invasion, and metastasis. Cell 2006; 124: 263-266.

24. Coffelt SB, Hughes R, Lewis CE. Tumor-associated macrophages: effectors of angiogenesis and tumor progression. Biochim Biophys Acta 2009; 1796: 11-18.

25. Lee CH, Espinosa I, Vrijaldenhoven S, Subramanian S, Montgomery KD, Zhu S et al. Prognostic significance of macrophage infiltration in leiomyosarcomas. Clin Cancer Res 2008; 14: 1423-1430.

26. Jensen TO, Schmidt H, Moller HJ, Hoyer M, Maniecki MB, Sjoegren P et al. Macrophage markers in serum and tumor have prognostic impact in American Joint Committee on Cancer stage I/II melanoma. J Clin Oncol 2009; 27: 3330-3337.

27. Steidl C, Lee T, Shah SP, Farinha P, Han G, Nayar T et al. Tumor-associated macrophages and survival in classic Hodgkin's lymphoma. N Engl J Med 2010; 362: 875-885.

28. Chung FT, Lee KY, Wang CW, Heh CC, Chan YF, Chen HW et al. Tumor-associated macrophages correlate with response to epidermal growth factor receptor-tyrosine kinase inhibitors in advanced non-small cell lung cancer. Int J Cancer 2012; 131 E227-E235.

29. Mantovani A, Allavena P. The interaction of anticancer therapies with tumor-associated macrophages. J Exp Med 2015; 212: 435-445.

30. De Palma M, Lewis CE. Macrophage regulation of tumor responses to anticancer therapies. Cancer Cell 2013; 23: 277-286.

31. Burma S, Chen BP, Murphy M, Kurimasa A, Chen DJ. ATM phosphorylates histone H2AX in response to DNA double-strand breaks. J Biol Chem 2001; 276: 42462-42467.
32. Murray PJ, Allen JE, Biswas SK, Fisher EA, Gilroy DW, Goerdt S et al. Macrophage activation and polarization: nomenclature and experimental guidelines. Immunity 2014; 41 : 14-20.

33. Guo Z, Kozlov S, Lavin MF, Person MD, Paull TT. ATM activation by oxidative stress. Science 2010; 330: 517-521.

34. Zhang Y, Choksi S, Chen K, Pobezinskaya Y, Linnoila I, Liu ZG. ROS play a critical role in the differentiation of alternatively activated macrophages and the occurrence of tumorassociated macrophages. Cell Res 2013; 23: 898-914.

35. Bedard K, Krause KH. The NOX family of ROS-generating NADPH oxidases: physiology and pathophysiology. Physiol Rev 2007; 87: 245-313.

36. Lambeth JD. NOX enzymes and the biology of reactive oxygen. Nat Rev Immunol 2004; 4: 181-189.

37. Trakarnsanga A, Ithimakin S, Weiser MR. Treatment of locally advanced rectal cancer: controversies and questions. World J Gastroenterol 2012; 18: 5521-5532.

38. Galli SJ, Borregaard N, Wynn TA. Phenotypic and functional plasticity of cells of innate immunity: macrophages, mast cells and neutrophils. Nat Immunol 2011; 12: 1035-1044.

39. Mantovani A, Locati M. Tumor-associated macrophages as a paradigm of macrophage plasticity, diversity, and polarization: lessons and open questions. Arterioscler Thromb Vasc Biol 2013; 33: 1478-1483.

40. Georgoudaki AM, Prokopec KE, Boura VF, Hellqvist E, Sohn S, Ostling J et al. Reprogramming tumor-associated macrophages by antibody targeting inhibits cancer progression and metastasis. Cell Rep 2016; 15: 2000-2011.

41. Tan HY, Wang N, Man K, Tsao SW, Che CM, Feng Y. Autophagy-induced RelB/p52 activation mediates tumour-associated macrophage repolarisation and suppression of hepatocellular carcinoma by natural compound baicalin. Cell Death Dis 2015; 6: e1942.

42. Rolny C, Mazzone M, Tugues S, Laoui D, Johansson I, Coulon C et al. HRG inhibits tumor growth and metastasis by inducing macrophage polarization and vessel normalization through downregulation of PIGF. Cancer Cell 2011; 19: 31-44.

43. Buhtoiarov IN, Sondel PM, Wigginton JM, Buhtoiarova TN, Yanke EM, Mahvi DA et al. Antitumour synergy of cytotoxic chemotherapy and anti-CD40 plus CpG-ODN immunotherapy through repolarization of tumour-associated macrophages. Immunology 2011; 132: 226-239.

44. Coscia M, Quaglino E, lezzi M, Curcio C, Pantaleoni F, Riganti C et al. Zoledronic acid repolarizes tumour-associated macrophages and inhibits mammary carcinogenesis by targeting the mevalonate pathway. J Cell Mol Med 2010; 14: 2803-2815.

45. Saliba DG, Heger A, Eames HL, Oikonomopoulos S, Teixeira A, Blazek K et al. IRF5:RelA interaction targets inflammatory genes in macrophages. Cell Rep 2014; 8: 1308-1317.

46. Bowdridge S, Gause WC. Regulation of alternative macrophage activation by chromatin remodeling. Nat Immunol 2010; 11: 879-881.

47. Krausgruber T, Saliba D, Ryzhakov G, Lanfrancotti A, Blazek K, Udalova IA. IRF5 is required for late-phase TNF secretion by human dendritic cells. Blood 2010; 115: 4421-4430.

48. Lien C, Fang CM, Huso D, Livak F, Lu R, Pitha PM. Critical role of IRF-5 in regulation of B-cell differentiation. Proc Natl Acad Sci USA 2010; 107: 4664-4668.

49. Jia X, Hu M, Lin Q, Ren H. Association of the IRF5 rs2004640 polymorphism with rheumatoid arthritis: a meta-analysis. Rheumatol Int 2013; 33: 2757-2761.

50. Graham RR, Kozyrev SV, Baechler EC, Reddy MV, Plenge RM, Bauer JW et al. A common haplotype of interferon regulatory factor 5 (IRF5) regulates splicing and expression and is associated with increased risk of systemic lupus erythematosus. Nat Genet 2006; 38: 550-555.

51. Carmona FD, Martin JE, Beretta L, Simeon CP, Carreira PE, Callejas JL et al. The systemic lupus erythematosus IRF5 risk haplotype is associated with systemic sclerosis. PLOS ONE 2013; 8: e54419.

52. Baskar R, Lee KA, Yeo R, Yeoh KW. Cancer and radiation therapy: current advances and future directions. Int J Med Sci 2012; 9: 193-199.

53. Eriksson D, Stigbrand T. Radiation-induced cell death mechanisms. Tumour Biol 2010; 31: 363-372.

54. Matei IR, Guidos CJ, Danska JS. ATM-dependent DNA damage surveillance in T-cell development and leukemogenesis: the DSB connection. Immunol Rev 2006; 209: 142-158.

55. So EY, Ouchi T. Translational initiation regulated by ATM in dendritic cells development. Cell Death Dis 2014; 5: e1418.

56. So EY, Kozicki M, Ouchi T. Roles of DNA damage response proteins in mitogen-induced Thp-1 differentiation into macrophage. J Cancer Biol Res 2013; 1: 1-3.

57. Hartlova A, Erttmann SF, Raffi FA, Schmalz AM, Resch U, Anugula S et al. DNA damage primes the type I interferon system via the cytosolic DNA sensor STING to promote antimicrobial innate immunity. Immunity 2015; 42: 332-343.

58. Pereira-Lopes S, Tur J, Calatayud-Subias JA, Lloberas J, Stracker TH, Celada A. NBS1 is required for macrophage homeostasis and functional activity in mice. Blood 2015; 126 : 2502-2510.

59. Allouch A, David A, Amie SM, Lahouassa H, Chartier L, Margottin-Goguet F et al. p21mediated RNR2 repression restricts HIV-1 replication in macrophages by inhibiting dNTP biosynthesis pathway. Proc Natl Acad Sci USA 2013; 110: E3997-E4006.

60. Solier S, Ryan MC, Martin SE, Varma S, Kohn KW, Liu H et al. Transcription poisoning by topoisomerase I is controlled by gene length, splice sites, and miR-142-3p. Cancer Res 2013; 73: 4830-4839. 